\title{
EASY LAMBDA-TERMS ARE NOT ALWAYS SIMPLE
}

\author{
Alberto Carraro $^{1,2}$ and Antonino Salibra ${ }^{1}$
}

\begin{abstract}
A closed $\lambda$-term $M$ is easy if, for any other closed term $N$, the lambda theory generated by $M=N$ is consistent. Recently, it has been introduced a general technique to prove the easiness of $\lambda$ terms through the semantical notion of simple easiness. Simple easiness implies easiness and allows to prove consistency results via construction of suitable filter models of $\lambda$-calculus living in the category of complete partial orderings: given a simple easy term $M$ and an arbitrary closed term $N$, it is possible to build (in a canonical way) a non-trivial filter model which equates the interpretation of $M$ and $N$. The question whether easiness implies simple easiness constitutes Problem 19 in the TLCA list of open problems. In this paper we negatively answer the question providing a non-empty co-r.e. (complement of a recursively enumerable) set of easy, but not simple easy, $\lambda$-terms.
\end{abstract}

1991 Mathematics Subject Classification. 03B40.

\section{INTRODUCTION}

Lambda theories are congruences on the set of lambda-terms which contain $\beta$-conversion. Lambda theories arise by syntactical or by semantic considerations. Indeed, a $\lambda$-theory may correspond to a possible operational semantics of $\lambda$-calculus, as well as it may be induced by a model of $\lambda$-calculus through the kernel congruence relation of the interpretation function. Lambda calculus has been originally investigated by using mainly syntactical methods (see Barendregt's book [7]). Syntactical proofs of consistency of remarkable $\lambda$-theories (for example, the theory equating all unsolvable $\lambda$-terms) were given in Barendregt's thesis [6]. Many other interesting examples of consistent $\lambda$-theories are studied in [7, Ch. 16,17], most of the time syntactically.

Keywords and phrases: Lambda calculus, easy lambda-terms, simple easy lambda-terms, filter models, ris models

1 DAIS, Università Ca' Foscari Venezia, Italia, \{acarraro, salibra\}@dsi.unive.it

2 PPS, Université Paris Diderot, France 
Since syntactic techniques are usually difficult to use in the study of $\lambda$-theories, semantical methods have been extensively investigated. After the first model, found by Scott in 1969 in the category of complete lattices and Scott continuous functions, a large number of mathematical models for $\lambda$-calculus, arising from syntax-free constructions, have been introduced in various Cartesian closed categories (ccc, for short) of domains and were classified into semantics according to the nature of their representable functions, see e.g. [7,12,37]. Scott continuous semantics [39] is the class of reflexive cpo-models, that are reflexive objects in the category Cpo whose objects are complete partial orders and morphisms are Scott continuous functions. The stable semantics (Berry [16]) and the strongly stable semantics (Bucciarelli-Ehrhard [18]) are refinements of the continuous semantics, introduced to approximate the notion of "sequential" Scott continuous function. Although Scott continuous semantics and the other mentioned semantics are structurally and equationally rich (each of them has $2^{\aleph_{0}}$ models inducing pairwise distinct $\lambda$-theories, see Kerth $[31,32]$ ), nevertheless, they do not match all possible operational semantics of $\lambda$-calculus, because there is a continuum of $\lambda$-theories which are omitted by all ordered models of $\lambda$-calculus with a bottom element (see Honsell-Ronchi [25]; Salibra [38]).

Some of the models in the above semantics, called webbed models, are built from lower level structures called "webs" (see Berline [12] for an extensive survey). The simplest class of webbed models is the class of graph models, which was isolated in the seventies by Plotkin, Scott and Engeler [23,37,40] within the continuous semantics. The class of graph models contains the simplest models of $\lambda$-calculus, is itself the easiest describable class, and represents nevertheless a continuum of (nonextensional) $\lambda$-theories. Another example of a class of webbed models, and the most established one, is the class of filter models. It was isolated at the beginning of the eighties by Barendregt, Coppo and Dezani [8], after the introduction of the intersection type discipline by Coppo and Dezani [20]. Not all filter models live in Scott continuous semantics: for example some of them lack the property of representing all continuous functions, and others were introduced for the stable semantics (see Honsell-Ronchi [24], Bastonero et al. [9]).

According to Jacopini [27] a closed $\lambda$-term $M$ is easy if, for any other closed term $N$, the $\lambda$-theory generated by the equality $M=N$ is consistent. Easy terms can be considered computational processes of a completely non-informative kind. Thus they are suitable candidates for representing inside $\lambda$-calculus the undefined value of a partial recursive function. The paradigmatic unsolvable term $\Omega \equiv(\lambda x . x x)(\lambda x . x x)$ was shown easy by Jacopini [27] (cf. [7, p. 402]) by a syntactic proof. Other syntactical proofs that certain terms are easy may be found in the literature, e.g., (Jacopini-Venturini-Zilli [28,29]; Intrigila [26]; Berarducci-Intrigila [11]; Kuper [34]).

Baeten and Boerboom gave in [5] the first semantical proof of the easiness of $\Omega$ by showing that, for all closed terms $M$ one can build a graph model satisfying the equation $\Omega=M$. Baeten and Boerboom build their graph model by a method of "forcing", which, although much simpler than the forcing techniques used in set theory, is somewhat in the same spirit. Forcing considerations have been extended 
by Zylberajch [44] to prove the simultaneous easiness of the members of some infinite family of easy terms (see also Berline-Salibra [15] and Berarducci [10]). However, the semantical methods via graph models have concrete limitations. For example, no semantical proof of the easiness of $\omega_{3} \omega_{3} \mathbf{I}$ (where $\omega_{3} \equiv \lambda x . x x x$ and $\mathbf{I} \equiv \lambda x . x)$ via graph models can exist, in contrast to the case $\Omega$, since Kerth [30] has shown that no graph model satisfies the identity $\omega_{3} \omega_{3} \mathbf{I}=\mathbf{I}$. Easiness of the term $\omega_{3} \omega_{3} \mathbf{I}$ was proved syntactically in (Jacopini-Venturini-Zilli [29]), but was only given a semantic proof in (Alessi et al. [2]), where the authors build, for each closed term $M$, a filter model of $\omega_{3} \omega_{3} \mathbf{I}=M$.

Alessi and Lusin in [4] introduced a general technique to prove the easiness of $\lambda$-terms through the notion of simple easiness. This notion implies easiness and can be handled in a natural way by semantic tools. It allows to prove consistency results via construction of suitable filter models of $\lambda$-calculus living in the category Cpo: given a simple easy term $M$ and an arbitrary closed term $N$, it is possible to build (in a canonical way) a non-trivial filter model which equates the interpretation of $M$ and $N$. In [3] Alessi, Dezani and Lusin prove in such a way the easiness of several terms. Moreover, simple easiness is interesting in itself, since it has to do with minimal sets of axioms which are needed in order to assign certain types to easy terms.

The TLCA list of open problems is a list of twenty-two problems that aims at collecting unresolved questions in the subject areas of the TLCA (Typed Lambda Calculi and Applications) series of conferences. Problem 19 in the TLCA list was posed by Fabio Alessi and Mariangiola Dezani-Ciancaglini in 2002 (see [1]) and asks whether easiness implies simple easiness. In this paper we negatively answer the question, providing a non-empty co-r.e. (i.e. it is the complement of a recursively enumerable set) set of easy, but non simple easy, $\lambda$-terms.

Outline of the proof. The main idea is to apply computability theory in the context of the models of $\lambda$-calculus and to consider the order theory $\operatorname{Ord}(\mathbf{A})=$ $\left\{(M, N): \llbracket M \rrbracket^{\mathbf{A}} \leq_{\mathbf{A}} \llbracket N \rrbracket^{\mathbf{A}}\right\}$ of a partially ordered model $\mathbf{A}$, as was done in [14]. The key step for the proof is the construction of a partially ordered model $\mathbf{P}$ with the following properties:

(i) $\operatorname{Ord}(\mathbf{P}) \subseteq \operatorname{Ord}(\mathbf{F})$, for every filter model $\mathbf{F}$ that lives in $\mathbf{C p o}$;

(ii) the set of closed $\lambda$-terms $N$ such that $\llbracket N \rrbracket^{\mathbf{P}} \leq \llbracket \lambda x . x \rrbracket^{\mathbf{P}}$ is co-r.e. .

We now briefly explain how such properties are obtained by our construction.

First of all we observe that for any filter model $\mathbf{F}$ in $\mathbf{C p o}$ and any inequality $M \sqsubseteq N$ which fails in $\mathbf{F}$, i.e. , $\llbracket M \rrbracket^{\mathbf{F}} \not_{\mathbf{F}} \llbracket N \rrbracket^{\mathbf{F}}$ there is a finite piece of $\mathbf{F}$ which is responsible for this failure. To such finite piece, let's say $F_{0}$, which is just a partial model of $\lambda$-calculus rather than an actual one, we apply a completion procedure whose outcome is a model $\mathbf{F}_{\omega}$ of $\lambda$-calculus such that $M \sqsubseteq N$ still fails in $\mathbf{F}_{\omega}$. Now $\mathbf{P}$ is defined as the direct product of the completions of all finite pieces of filter models; as a direct product of models, $\mathbf{P}$ itself is a model and by construction every inequality which holds in $\mathbf{P}$ also holds in every filter model in $\mathbf{C p o}$. This explains property (i). 
The completion procedure that we use is also effective and the web of the completion $\mathbf{F}_{\omega}$ of a finite piece $F_{0}$ of a filter model admits a numeration and has effectivity properties that allow to prove that the set of closed $\lambda$-terms $N$ such that

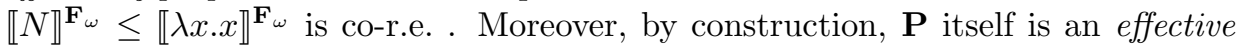
product of effective structures, so that it is possible to semi-decide the set of closed

$\lambda$-terms whose interpretation in $\mathbf{P}$ is not below $\llbracket \lambda x . x \rrbracket^{\mathbf{P}}$. This roughly explains property (ii).

With these properties at hand we are now in the position of exhibiting a nonempty set of easy but non-simple easy terms.

By property (ii) the set $X=\left\{N: N\right.$ closed and $\left.\llbracket N \rrbracket^{\mathbf{P}} \leq \llbracket \lambda x . x \rrbracket^{\mathbf{P}}\right\}$ is a nonempty beta-closed co-r.e. set of $\lambda$-terms. Moreover, the set $E$ of all easy terms is also beta-closed and co-r.e. ; now a theorem of Visser [43] allows us to say that $E \cap X$ is co-r.e. and non-empty too. Finally, using property (i) we can prove that the assumption of simple easiness for a term in $E \cap X$ leads to the contradiction of Böhm's Theorem [17], so that the set $E \cap X$ witnesses the existence of easy but non-simple easy terms.

\section{Preliminaries}

If $A$ is a set, then we denote by $\mathcal{P}(A)$ the power set of $A$ and by $\mathcal{P}_{\mathrm{f}}(A)$ the set of all finite subsets of $A$. We write $a \subseteq_{\mathrm{f}} A$ for $a \subseteq A$ and $a$ is finite. If $f: A \rightarrow B$ is a function, and $a \subseteq A$, then we define $f(a)=\{f(\alpha): \alpha \in a\}$.

We denote by $\mathbb{N}$ the set of natural numbers. A set $X \subseteq \mathbb{N}$ is r.e. if it is the domain of a partial recursive function. The complement of an r.e. set is called a co-r.e. set. If both $X$ and its complement are r.e., $X$ is called decidable.

Let $(D, \leq)$ be a poset. A subset $X \subseteq D$ is directed if, for all $u, v \in X$, there exists $z \in X$ such that $u \leq z$ and $v \leq z$. A poset $D$ is a complete partial order (cpo, for short) if it has a least element (denoted by $\perp_{D}$ ) and every directed set $X \subseteq D$ admits a least upper bound (denoted by $\sqcup X)$. If $D$ is a cpo, then $[D \rightarrow D]$ denotes the cpo of Scott continuous functions from $D$ to $D$ ordered pointwise.

An element $d$ of a cpo $D$ is called compact if for every directed $X \subseteq D$ we have that $d \leq \sqcup X$ implies $d \leq e$ for some $e \in X$. We write $\mathcal{K}(D)$ for the collection of compact elements of $D$.

An algebraic cpo $D$ is a cpo such that for every $x \in D$ the set $\{d \in \mathcal{K}(D): d \leq x\}$ is directed and $x$ is its least upper bound. An algebraic lattice is a complete lattice which is an algebraic cpo.

Given two objects $A, B$ of a category $\mathbf{C}$, the set of all morphisms $f: A \rightarrow B$ is denoted by $\mathbf{C}(A, B)$. A category $\mathbf{C}$ is Cartesian if, and only if, it has the terminal object $\top$ and finite products $A \times B$ of all objects $A, B$. More precisely for all objects $A, B$ one has a natural isomorphism $\mathbf{C}(A, B \times C) \cong \mathbf{C}(A, B) \times \mathbf{C}(A, C)$ obtained via the projections $\pi_{1}, \pi_{2}$ and the pairing operation $\langle\cdot, \cdot\rangle$ that given two morphisms $f: A \rightarrow B, g: A \rightarrow C$ returns a morphism $\langle f, g\rangle: A \rightarrow B \times C$. The product of morphisms $f: A \rightarrow B$ and $g: A^{\prime} \rightarrow B^{\prime}$ is given by $f \times g=$ $\left\langle f \circ \pi_{1}, g \circ \pi_{2}\right\rangle: A \times A^{\prime} \rightarrow B \times B^{\prime}$. 
The set of points of an object $A$ is $\mathbf{C}(\top, A) ; A$ has enough points if for all $f, g \in \mathbf{C}(A, A)$ such that $f \neq g$ there exists a point $p$ of $A$ such that $f \circ p \neq g \circ p$. A category has enough points if each of its objects has enough points.

A Cartesian closed category (ccc, for short) is a Cartesian category with exponents: that is, a category in which the collection of morphisms between two objects can be internalized as an object itself. In order to be precise we report explicitly the data and the equations they must satisfy.

Definition 1.1. A category $\mathbf{C}$ is a ccc iff it is Cartesian and for all objects $A, B, C$ there exist an object $B^{A}$, a morphism ev : $B^{A} \times A \rightarrow B$ and a map cur : $\mathbf{C}(C \times A, B) \rightarrow \mathbf{C}\left(C, B^{A}\right)$ which satisfy:

$$
\mathrm{ev} \circ(\operatorname{cur}(f) \times \mathrm{id})=f \quad \operatorname{cur}(\mathrm{ev} \circ(g \times \mathrm{id}))=g
$$

for all $f: C \times A \rightarrow B, g: D \rightarrow B^{A}$.

The category Cpo of cpos and Scott continuous functions is a ccc with enough points. By ALat we denote the full subcategory of Cpo determined by the algebraic lattices; ALat is a ccc too. Then the points of a cpo $\mathcal{D}$ are exactly the elements of its underlying set and the space $\mathbf{C p o}(\mathcal{D}, \mathcal{E})$ of morphisms is the same thing as the exponent $\mathcal{E}^{\mathcal{D}}$, i.e. the space $[\mathcal{D} \rightarrow \mathcal{E}]$ of Scott continuous functions. Evaluation morphisms are defined by ev $(f)(x)=f(x)$.

\section{2. $\lambda$-CALCULUS AND $\lambda$-THEORIES}

With regard to the $\lambda$-calculus we follow the notation and terminology of [7]. $\Lambda$ and $\Lambda^{o}$ are, respectively, the set of $\lambda$-terms and of closed $\lambda$-terms. We denote $\alpha \beta$ conversion by $\lambda \beta$. A $\lambda$-theory is a congruence on $\Lambda$ (with respect to the operators of abstraction and application) which contains $\lambda \beta$. A $\lambda$-theory is consistent if it does not equate all $\lambda$-terms, inconsistent otherwise. The set of $\lambda$-theories constitutes a complete lattice w.r.t. inclusion, whose top is the inconsistent $\lambda$-theory and whose bottom is the theory $\lambda \beta$. The $\lambda$-theory generated by a set $X$ of identities is the intersection of all $\lambda$-theories containing $X$.

A $\lambda$-term $M \in \Lambda^{o}$ is solvable if it has a head normal form, i.e., $M$ is $\beta$-convertible to a term of the form $\lambda \vec{x} \cdot y \vec{N}$. A $\lambda$-term $M \in \Lambda^{o}$ is unsolvable if it is not solvable. Unsolvable terms have been regarded to as representing undefined computations.

A $\lambda$-term $M \in \Lambda^{o}$ is easy if, for every other term $N \in \Lambda^{\circ}$, the lambda theory generated by the identity $M=N$ is consistent. Every easy term is unsolvable.

Of great relevance for the results of the present paper is a theorem of Albert Visser [43]; he originally formulated his result in topological terms, but we prefer to report here a rephrased version of his statement to avoid reference to unnecessary notions. Just recall that a set of $\lambda$-terms is $\beta$-closed if it is the union of $\beta$ equivalence classes.

Theorem 2.1 (Visser [43]). The intersection of two non-empty $\beta$-closed co-r.e. sets of $\lambda$-terms is non-empty and co-r.e. . 
Lemma 2.2. (i) The set $E$ of all easy $\lambda$-terms is non-empty, $\beta$-closed, and co-r.e.

(ii) Every non-trivial, co-r.e. and $\beta$-closed subset of $\Lambda$ contains a non-empty co-r.e. set of easy $\lambda$-terms.

Proof. (i) Clearly $E$ is non-empty because it contains the term $\Omega=(\lambda x . x x)(\lambda x . x x)$, and it is $\beta$-closed because if $N$ is $\beta$-equivalent to an easy $\lambda$-term $M$, then $N$ itself can be consistently equated to any other closed $\lambda$-term. A closed $\lambda$-term $M$ is not easy if, and only if, there exists a closed $\lambda$-term $N$ such that the $\lambda$-theory generated by the identity $M=N$ contains the identity $\lambda x y \cdot x=\lambda x y \cdot y$ (condition equivalent to inconsistency). Therefore $E$ is also co-r.e. .

(ii) Follows immediately from Theorem 2.1.

Another milestone that we use for our final proof is Böhm's Theorem. More specifically, we will make use of an easy consequence of it, namely the incomparability of closed distinct $\beta \eta$-normal forms in some partially ordered models of $\lambda$-calculus.

Theorem 2.3 (Böhm [17]). If $M, N$ are two closed distinct $\beta \eta$-normal forms, then for all $\lambda$-terms $P, Q$ there exists a sequence $\vec{L}$ of $\lambda$-terms such that $M \vec{L}={ }_{\beta} P$ and $N \vec{L}={ }_{\beta} Q$.

\subsection{Models of LAMBda-CALCUlus: $\lambda$-MOdels AND REFLEXIVE OBJeCtS IN CCC'S}

It took some time, after Scott gave his model construction, for consensus to arise on the general notion of a model of the $\lambda$-calculus. There are mainly two descriptions that one can give: the category-theoretical and the algebraic one. Besides the different languages in which they are formulated, the two approaches are intimately connected (see Koymans [33]). The categorical notion of model is well-suited for constructing concrete models, while the algebraic one is rather used to understand global properties of models (constructions of new models out of existing ones, closure properties, etc.) and to obtain results about the structure of the lattice of $\lambda$-theories.

\subsection{1. $\lambda$-models}

The algebraic description of models of $\lambda$-calculus proposes two kinds of structures, viz. the $\lambda$-algebras and the $\lambda$-models, both based on the notion of combinatory algebra. We will focus on $\lambda$-models.

A combinatory algebra $\mathbf{A}=(A, \cdot, K, S)$ is a structure with a binary operation called application and two distinguished elements $K$ and $S$ called basic combinators. The symbol "." is usually omitted from expressions and by convention application associates to the left, allowing to leave out superfluous parentheses. The class of combinatory algebras is axiomatized by the equations $K x y=x$ and $S x y z=x z(y z)$. Intuitively elements on the left-hand side of an application are to be seen as functions operating on arguments, placed on the right-hand side. 
Hence it is natural to say that a function $f: A \rightarrow A$ is representable (in $\mathbf{A}$ ) if there exists an element $a \in A$ such that $f(b)=a b$ for all $b \in A$. For example the identity function is represented by the combinator $I=S K K$.

Let $\operatorname{Env}_{A}$ be the set of $A$-environments, i.e., the functions from the set Var of $\lambda$-calculus variables to $A$. For every $x \in \operatorname{Var}$ and $a \in A$ we denote by $\rho[x:=a]$ the environment $\rho^{\prime}$ which coincides with $\rho$ everywhere except on $x$, where $\rho^{\prime}$ takes the value $a$. The aim is to define an interpretation $\llbracket \cdot \rrbracket^{\mathbf{A}}: \Lambda \times \operatorname{Env}_{A} \rightarrow A$ of $\lambda$-terms by structural induction in such a way that:

$$
\llbracket x \rrbracket_{\rho}^{\mathbf{A}}=\rho(x) \quad \text { and } \quad \llbracket M N \rrbracket_{\rho}^{\mathbf{A}}=\llbracket M \rrbracket_{\rho}^{\mathbf{A}} \llbracket N \rrbracket_{\rho}^{\mathbf{A}} .
$$

Concerning the definition of $\llbracket \lambda x . M \rrbracket_{\rho}^{\mathbf{A}}$, one would like to set it equal to an element $b \in A$ representing the function $f(a)=\llbracket M \rrbracket_{\rho[x:=a]}^{\mathbf{A}}$.

Clearly this "definition" is not acceptable unless one is sure that $f$ is representable and that there is a canonical way of identifying an element that represents $f$.

The axioms of an elementary subclass of combinatory algebras, called $\lambda$-models, were expressly chosen to make coherent the previous definition of interpretation (see Meyer [36], Scott [41], Barendregt [7, Def. 5.2.7]). In addition to five axioms due to Curry (see [7, Thm. 5.2.5]), the Meyer-Scott axiom is the most important one in the definition of a $\lambda$-model. In the first-order language of combinatory algebras it takes the following form

$$
\forall x y \cdot(\forall z \cdot x z=y z) \Rightarrow 1 x=1 y
$$

where the combinator $1=S(K I)$ is made into an inner choice operator. Indeed, given any $a$, the element $1 a$ represents the same function as $a$; by Meyer-Scott axiom, $1 c=1 d$ for all $c, d$ representing the same function.

Let $R(A)$ be the set of representable functions. When $\mathbf{A}$ is a $\lambda$-model it is possible to prove that the two functions

$$
\begin{array}{lll}
F: A \rightarrow R(A) & G: R(A) \rightarrow A \\
a \mapsto(b \mapsto a b) & f \mapsto 1 a \quad \text { where } a \text { represents } f
\end{array}
$$

are such that $F \circ G=\mathrm{id}_{R(A)}$ and the interpretation

$\llbracket x \rrbracket_{\rho}^{\mathbf{A}}=\rho(x) ; \quad \llbracket M N \rrbracket_{\rho}^{\mathbf{A}}=F\left(\llbracket M \rrbracket_{\rho}^{\mathbf{A}}\right)\left(\llbracket N \rrbracket_{\rho}^{\mathbf{A}}\right) ; \quad \llbracket \lambda x \cdot M \rrbracket_{\rho}^{\mathbf{A}}=G\left(a \in A \mapsto \llbracket M \rrbracket_{\rho[x:=a \rrbracket}^{\mathbf{A}}\right)$

is well-defined, since each function $f(a)=\llbracket M \rrbracket_{\rho[x:=a]}^{\mathbf{A}}$ is representable. This is the kind of interpretation we will refer to.

Each $\lambda$-model $\mathbf{A}$ induces a $\lambda$-theory, denoted here by $\operatorname{Eq}(\mathbf{A})$, and called the equational theory of $\mathbf{A}$. Thus, $M=N \in \mathrm{Eq}(\mathbf{A})$ if, and only if, $M$ and $N$ have the same interpretation in $\mathbf{A}$. If moreover $\mathbf{A}$ is endowed with a partial order $\leq$, which is compatible with application, then $\mathbf{A}$ also induces an order theory $\operatorname{Ord}(\mathbf{A})=\left\{(M, N): \llbracket M \rrbracket_{\rho}^{\mathbf{A}} \leq \llbracket N \rrbracket_{\rho}^{\mathbf{A}}\right.$ for all environments $\left.\rho\right\}$. We write $M \sqsubseteq_{\mathbf{A}} N$ or $M \sqsubseteq N$ for $(M, N) \in \operatorname{Ord}(\mathbf{A})$. 
If $M$ is a closed $\lambda$-term we write $\llbracket M \rrbracket^{\mathbf{A}}$ for $\llbracket M \rrbracket_{\rho}^{\mathbf{A}}$, since the interpretation of closed $\lambda$-terms does not depend on the environment.

In conclusion the $\lambda$-models can be described by first-order axioms, but not by equations only. Indeed the class of $\lambda$-models is not closed under substructures nor under homomorphic images but it is closed under direct products.

\subsubsection{Reflexive objects in ccc's}

Let $\mathbf{C}$ be a category. Then a pair $(F, G)$ is a retraction pair from an object $A$ into an object $B$ if $F: B \rightarrow A$ and $G: A \rightarrow B$ are two morphisms satisfying $F \circ G=\mathrm{id}_{A}$. The categorical description of a model of $\lambda$-calculus consists of a ccc C together with a triple $(U, F, G)$ such that $(F, G)$ is a retraction pair from $U^{U}$ into $U$ : one such object $U$ is called a reflexive object.

For the sake of our paper, we rely on a theorem of Koymans [33] which says that if $\mathbf{U}=(U, F, G)$ is a reflexive object with enough points in a ccc, then the set $\mathbf{C}(\top, U)$ of morphisms from the terminal object to $U$ can be endowed with the structure of a $\lambda$-model where application is given by $x \cdot y=\mathrm{ev} \circ\langle F \circ x, y\rangle$ and the basic combinators are suitable points of $U$.

Let $\mathbf{D}=(\mathcal{D}, F, G)$ be a reflexive object in $\mathbf{C p o}$. Then the points of $\mathbf{D}$ are exactly the elements of the underlying set of $\mathcal{D}$ and the space of morphisms $\operatorname{Cpo}(\mathcal{D}, \mathcal{D})$ is the space $[\mathcal{D} \rightarrow \mathcal{D}$ ] of Scott continuous endofunctions. Since $\mathbf{C p o}$ has enough points, the $\lambda$-terms are interpreted as elements of $\mathbf{D}$.

\subsection{Filter models}

In this section we introduce the class of filter models of $\lambda$-calculus, first introduced by Coppo et al. in [21].

We remark that a filter model $\mathbf{F}$ lives in $\mathbf{C p o}$ if $\mathbf{F}$ is a reflexive algebraic lattice: this implies that all continuous endo-functions are representable in $\mathbf{F}$, viewed as a $\lambda$-model. Not all filter models live in $\mathbf{C p o}$, as some of them fail to represent all continuous functions: such failure (or non-failure) for a filter model $\mathbf{F}$ depends on the properties of the extended abstract type structure on which $\mathbf{F}$ is built.

Definition 2.4. (Coppo et al. [21, Def. 1.1]) An extended abstract type structure (eats, for short) is a structure $\mathcal{S}=(S, \leq, \wedge, \rightarrow, \omega)$, where $S$ is a set, $\omega \in S, \wedge$ and $\rightarrow$ are binary functions over $S$ and $\leq$ is a binary relation on $S$, satisfying the following axioms and rules:

$$
\begin{array}{lll}
\overline{\alpha \leq \omega} & \overline{\omega \leq \omega \rightarrow \omega} & \overline{(\alpha \rightarrow \beta) \wedge\left(\alpha \rightarrow \beta^{\prime}\right) \leq \alpha \rightarrow\left(\beta \wedge \beta^{\prime}\right)} \\
\overline{\alpha \wedge \beta \leq \alpha} & \overline{\alpha \wedge \beta \leq \beta} & \overline{\alpha \leq \alpha \wedge \alpha} \\
\frac{\alpha \leq \alpha^{\prime} \quad \beta \leq \beta^{\prime}}{\alpha \wedge \beta \leq \alpha^{\prime} \wedge \beta^{\prime}} & \frac{\alpha^{\prime} \leq \alpha \quad \beta \leq \beta^{\prime}}{\alpha \rightarrow \beta \leq \alpha^{\prime} \rightarrow \beta^{\prime}} & \frac{\alpha \leq \alpha^{\prime} \quad \alpha^{\prime} \leq \beta}{\alpha \leq \beta}
\end{array}
$$


Observe that $\leq$ is a preorder on $S$. It is customary to define an equivalence relation $\sim$ on types as follows: $\alpha \sim \beta$ iff $\alpha \leq \beta \leq \alpha$. For example $\omega \sim \omega \rightarrow \omega$, $\alpha \sim \alpha$ for all $\alpha \in S$; the operator $\wedge$ is a meet operator modulo $\sim$.

A filter of an eats $\mathcal{S}$ is a non-empty subset $X \subseteq S$ which is upward closed w.r.t. $\leq$ and closed under $\wedge$; the filter generated by a subset $Y \subseteq S$ is the set $\uparrow Y=\left\{\alpha \in S: \exists \beta_{1}, \ldots, \beta_{n} \in Y . \beta_{1} \wedge \cdots \wedge \beta_{n} \leq \alpha\right\}$. By $\mathcal{F}_{\mathcal{S}}$ we denote the set of all filters of $\mathcal{S}$, which is an algebraic lattice with respect to set inclusion, and whose compact elements are the filters of the form $\uparrow\{\alpha\}$, for $\alpha \in S$.

Given $\mathcal{F}_{\mathcal{S}}$, it is possible to define the following two continuous maps $F: \mathcal{F}_{\mathcal{S}} \rightarrow$ $\left[\mathcal{F}_{\mathcal{S}} \rightarrow \mathcal{F}_{\mathcal{S}}\right]$ and $G:\left[\mathcal{F}_{\mathcal{S}} \rightarrow \mathcal{F}_{\mathcal{S}}\right] \rightarrow \mathcal{F}_{\mathcal{S}}:$

$F(X)(Y)=\{\beta \in S: \exists \alpha \in Y . \alpha \rightarrow \beta \in X\} ; \quad G(f)=\uparrow\{\alpha \rightarrow \beta \in S: \beta \in f(\uparrow \alpha)\}$.

However, the triple $\left(\mathcal{F}_{\mathcal{S}}, F, G\right)$ need not to be neither a reflexive object in the category ALat, nor a model of the $\lambda$-calculus at all. There is a condition, isolated in [21, Def. 2.12], that characterizes those eats $\mathcal{S}$ such that $\mathcal{F}_{\mathcal{S}}$ is a reflexive algebraic lattice: this is formalized in the forthcoming theorem.

Theorem 2.5 (Coppo et al. [21]). The structure $\left(\mathcal{F}_{\mathcal{S}}, F, G\right)$ is a reflexive algebraic lattice iff the following condition (C3) holds in $\mathcal{S}$, for all $\alpha_{i}, \beta_{i}, \gamma, \delta \in S$ :

(C3) if $\bigwedge_{i=1}^{n}\left(\alpha_{i} \rightarrow \beta_{i}\right) \leq \gamma \rightarrow \delta$, then either $\omega \leq \delta$ or $\exists J \subseteq\{1, \ldots, n\}$ such that $J \neq \emptyset, \gamma \leq \bigwedge_{j \in J} \alpha_{j}$ and $\bigwedge_{j \in J} \beta_{j} \leq \delta$

Proof. If $f \in\left[\mathcal{F}_{\mathcal{S}} \rightarrow \mathcal{F}_{\mathcal{S}}\right]$, then we have $F(G(f))(Y)=\{\beta \in S: \exists \alpha \in Y$. $\alpha \rightarrow \beta \in$ $\uparrow\{\alpha \rightarrow \beta \in S: \beta \in f(\uparrow \alpha)\}\}=f(Y)$ iff condition (C3) holds in $\mathcal{S}$.

\subsection{Simple EASY $\lambda$-TERMS}

Alessi and Lusin [4] isolated a subclass of filter models, generated by what they call easy intersection type systems, that they used to prove the easiness of some $\lambda$-terms. Roughly speaking they say that a $\lambda$-term $M$ is simple easy if for every closed $\lambda$-term $N$ there exists an easy intersection type system which generates a filter model satisfying the identity $M=N$.

An intersection type language $\mathbb{T}$ is a set of formulas, called types, built on a given set of constants by means of the type constructors " $\wedge$ " and " $\rightarrow$ ". The constant $\omega$ belongs to every intersection type language. The letter $\alpha, \beta, \gamma, \delta$ will range over constants (different from $\omega$ ), while $\sigma, \tau, \ldots$ over types. Whenever we write an expression like $\bigwedge_{i \in I} \alpha_{i}$, we will implicitly assume that the set $I$ is finite.

The concept of an easy intersection type theory over an intersection type language was defined for the first time in Alessi et al. [2, Def. 2] (see also [4, Def. 1.2]).

Definition 2.6 (Alessi-Lusin [2, Def. 2]). An easy intersection type theory (eitt, for short) over an intersection type language $\mathbb{T}$ is the set of inequalities of the form $\sigma \leq \tau(\sigma, \tau \in \mathbb{T})$ derivable from a collection $\mathcal{T}$ of axioms and rules such that: 
(1) $\mathcal{T}$ contains the axiom and the rules characterizing eats's (and no further rules);

(2) $\mathcal{T}$ may only contain additional axioms of the following two shapes: $\alpha \leq \beta$ or $\alpha \sim \bigwedge_{i \in I}\left(\gamma_{i} \rightarrow \tau_{i}\right)$, where $\alpha, \beta, \gamma_{i}$ are constants with $\alpha, \beta \not \equiv \omega$, and $\tau_{i} \in \mathbb{T}$

(3) for each constant $\alpha \not \equiv \omega$ there exists exactly one axiom of the shape $\alpha \sim$ $\bigwedge_{i \in I}\left(\gamma_{i} \rightarrow \tau_{i}\right)$

(4) if $\mathcal{T}$ contains $\alpha \sim \bigwedge_{i \in I}\left(\gamma_{i} \rightarrow \tau_{i}\right)$ and $\beta \sim \bigwedge_{j \in J}\left(\delta_{j} \rightarrow \sigma_{j}\right)$, then $\mathcal{T}$ contains also $\alpha \leq \beta$ iff, for each $j \in J$, there exists $i_{j} \in I$ such that $\delta_{j} \leq \gamma_{i_{j}}$ and $\tau_{i_{j}} \leq \sigma_{j}$.

We ambiguously denote by $\mathcal{T}$ the eitt generated by the set $\mathcal{T}$ of rules and axioms and we write $\sigma \leq_{\mathcal{T}} \tau$ to indicate that $\sigma \leq \tau$ is derivable from $\mathcal{T}$. The items (1)(4) of the above Definition 2.6 have different purposes: some of them are taken from [21] and allow the construction of a reflexive algebraic lattice out of a set of inequalities over an intersection type language, while others are proper to Alessi and Lusin [4] and concern technicalities of their constructions.

What is important for us is that the following theorem holds.

Theorem 2.7 ( [4]). Every eitt $\mathcal{T}$ is an eats that satisfies condition (C3) and hence $\left(\mathcal{F}_{\mathcal{T}}, F, G\right)$ is a reflexive algebraic lattice.

Let $\mathcal{T}, \mathcal{S}$ be eitt's over the type languages $\mathbb{T}$ and $\mathbb{S}$ respectively. We say that $\mathcal{S}$ is a conservative extension of $\mathcal{T}$, written $\mathcal{T} \sqsubseteq \mathcal{S}$, if $\mathbb{T} \subseteq \mathbb{S}$ and, for all $\tau, \sigma \in \mathbb{T}$, $\tau \leq_{\mathcal{T}} \sigma$ iff $\tau \leq_{\mathcal{S}} \sigma$.

Definition 2.8 (Alessi-Lusin [4]). An unsolvable term $M$ is simple easy if for every eitt $\mathcal{T}$ over the type language $\mathbb{T}$ and every type $\tau \in \mathbb{T}$ there exists a conservative extension $\mathcal{S}$ of $\mathcal{T}$ such that $\sigma \in \llbracket M \rrbracket^{\mathcal{F}_{\mathcal{S}}} \Longleftrightarrow \exists \sigma^{\prime} \in \llbracket M \rrbracket^{\mathcal{F}_{\mathcal{T}}} . \sigma^{\prime} \wedge \tau \leq_{\mathcal{S}} \sigma$, for all types $\sigma$ in the type language of $\mathcal{S}$.

Roughly speaking, Definition 2.8 says that given an arbitrary intersection type $\tau$, one can find a suitable pre-order on types which allows to derive $\tau$ for $M$.

Theorem 2.9 ( [4]). Given a fixed simple easy term $M$, for every closed $\lambda$-term $N$ there exists a non-trivial eitt $\mathcal{T}$, and thus a filter model $\mathcal{F}_{\mathcal{T}}$ living in $\mathbf{C p o}$, such that $\llbracket M \rrbracket^{\mathcal{F}_{\mathcal{T}}}=\llbracket N \rrbracket^{\mathcal{F}_{\mathcal{T}}}$.

We refer the reader to [4, Thm. 3.5] for the complete proof of Theorem 2.9 with detailed construction of the filter model $\mathcal{F}_{\mathcal{T}}$. The following corollary explains the importance of simple easiness.

Corollary 2.10. Every simple easy term is easy.

\section{INFORMATION SYSTEMS FOR ALGEBRAIC LATTICES}

Algebraic lattices have a representation as lattices of closed elements of closure operators. In this section we define closure operators through a suitable class of information systems. 
Information systems were introduced by Dana Scott in [42] to give an appealing and suggestive representation of Scott domains. An information system consists of a set of tokens endowed with an entailment relation and a consistency predicate. It determines a Scott domain whose elements are those sets of tokens which are consistent and closed with respect to the entailment relation; the ordering is just set inclusion. Vice versa a Scott domain defines an information system through its compact elements.

Warning. For the purposes of this paper, what we call information system is actually a minor modification of the original structure, expressive enough to represent all algebraic lattices. In particular we drop the consistency predicate from the original definition.

Definition 3.1. An information system is a pair $\mathcal{A}=\left(A, \vdash_{\mathcal{A}}\right)$, where $A$ is a non-empty set of tokens, and $\vdash_{\mathcal{A}} \subseteq \mathcal{P}_{\mathrm{f}}(A) \times \mathcal{P}_{\mathrm{f}}(A)$ is a reflexive transitive binary relation satisfying the following condition:

$$
a \vdash_{\mathcal{A}} b_{1}, a \vdash_{\mathcal{A}} b_{2}, c \supseteq_{\mathrm{f}} a \Rightarrow c \vdash_{\mathcal{A}} b_{1} \cup b_{2} .
$$

As a matter of notation, we write $a \vdash_{\mathcal{A}} \alpha$ for $a \vdash_{\mathcal{A}}\{\alpha\}$.

Notice that

(i) $a \vdash_{\mathcal{A}} b$ iff $a \vdash_{\mathcal{A}} \beta$ for all $\beta \in b$;

(ii) $a_{i} \vdash_{\mathcal{A}} b_{i}(i=1, \ldots n)$ imply $a_{1} \cup \cdots \cup a_{n} \vdash_{\mathcal{A}} b_{1} \cup \cdots \cup b_{n}$.

(iii) $a \vdash_{\mathcal{A}} \emptyset$

An algebraic closure operator is any map $\overline{(\cdot)}: \mathcal{P}(A) \rightarrow \mathcal{P}(A)$ satisfying the following conditions:

(i) $x \subseteq \bar{x}$;

(ii) $\overline{\bar{x}}=\bar{x}$;

(iii) $x \subseteq y \Rightarrow \bar{x} \subseteq \bar{y}$

(iv) $\bar{x}=\cup_{a \subseteq_{\mathrm{f}} x} \bar{a}$.

\section{Proposition 3.2.}

(i) Let $\mathcal{A}=\left(A, \vdash_{\mathcal{A}}\right)$ be an information system. Then, the function

$\overline{(\cdot)}_{\mathcal{A}}: \mathcal{P}(A) \rightarrow \mathcal{P}(A)$, defined by $\bar{x}_{\mathcal{A}}=\cup\left\{b: \exists a \subseteq_{\mathrm{f}} x . a \vdash_{\mathcal{A}} b\right\}$, is an algebraic closure operator.

(ii) Let $c: \mathcal{P}(A) \rightarrow \mathcal{P}(A)$ be an algebraic closure operator. Then, the pair $\mathcal{A}_{c}=\left(A, \vdash_{\mathcal{A}_{c}}\right)$, defined by $a \vdash_{\mathcal{A}_{c}} b$ iff $b \subseteq_{\mathrm{f}} c(a)$, is an information system.

(iii) The two transformations are inverses of each other.

A subset $x \subseteq A$ is closed if $\bar{x}_{\mathcal{A}}=x$. The set of all closed sets ordered by inclusion is an algebraic lattice, denoted by $\mathcal{A}^{+}$.

Proposition 3.3. Every algebraic lattice $L$ is isomorphic to the algebraic lattice of the closed elements of a suitable information system.

Proof. Define an information system $\mathcal{K}_{L}=\left(\mathcal{K}(L), \vdash_{\mathcal{K}_{L}}\right)$, where $\mathcal{K}(L)$ is the set of compact elements of $L$, and $a \vdash_{\mathcal{K}_{L}} b$ iff $\sqcup b \leq \sqcup a$. 
Definition 3.4. Let $\mathcal{A}=\left(A, \vdash_{\mathcal{A}}\right)$ be an information system. We define another information system $\mathcal{A} \Rightarrow \mathcal{A}=\left(\mathcal{P}_{\mathrm{f}}(A) \times \mathcal{P}_{\mathrm{f}}(A), \vdash_{\mathcal{A} \Rightarrow \mathcal{A}}\right)$ by setting:

$$
\left\{\left(a_{1}, b_{1}\right), \ldots,\left(a_{n}, b_{n}\right)\right\} \vdash_{\mathcal{A} \Rightarrow \mathcal{A}}(c, d) \text { iff } \bigcup\left\{b_{i}: c \vdash_{\mathcal{A}} a_{i}\right\} \vdash_{\mathcal{A}} d .
$$

The information system $\mathcal{A} \Rightarrow \mathcal{A}$ will be called the exponential of $\mathcal{A}$.

Proposition 3.5. The algebraic lattice $\left[\mathcal{A}^{+} \rightarrow \mathcal{A}^{+}\right]$of all Scott continuous functions from $\mathcal{A}^{+}$into $\mathcal{A}^{+}$is isomorphic (in the category ALat) to the algebraic lattice $(\mathcal{A} \Rightarrow \mathcal{A})^{+}$.

Proof. We define two continuous functions gph : $\left[\mathcal{A}^{+} \rightarrow \mathcal{A}^{+}\right] \rightarrow(\mathcal{A} \Rightarrow \mathcal{A})^{+}$and fun $:(\mathcal{A} \Rightarrow \mathcal{A})^{+} \rightarrow\left[\mathcal{A}^{+} \rightarrow \mathcal{A}^{+}\right]$as follows:

$$
\operatorname{gph}(f)=\left\{(a, b): b \subseteq_{\mathrm{f}} f\left(\bar{a}_{\mathcal{A}}\right)\right\} ; \quad \text { fun }(x)(y)=\bigcup\left\{b: \exists a \subseteq_{\mathrm{f}} y .(a, b) \in x\right\},
$$

for all continuous functions $f: \mathcal{A}^{+} \rightarrow \mathcal{A}^{+}$, all closed subsets $x \in(\mathcal{A} \Rightarrow \mathcal{A})^{+}$and all $y \in \mathcal{A}^{+}$. Then we have fun $(\operatorname{gph}(f))(y)=\bigcup\left\{b: \exists a \subseteq_{\mathrm{f}} y . b \subseteq_{\mathrm{f}} f\left(\bar{a}_{\mathcal{A}}\right)\right\}=f(y)$ and $\operatorname{gph}(f u n(x))=\left\{(a, b): \exists a^{\prime} \subseteq_{\mathrm{f}} \bar{a}_{\mathcal{A}} \cdot\left(a^{\prime}, b\right) \in x\right\}=x$.

\section{WeBBED MODELS OF $\lambda$-CALCULUS}

Some of the models of $\lambda$-calculus are called webbed models because they are built from lower level structures called "webs" (see Berline $[12,13]$ for an accurate survey). Typically a web is a set with additional structure and a webbed model is a partial order (usually a domain) whose elements are special subsets of the web. We now introduce a class of webbed models of $\lambda$-calculus arising from information systems that include the filter models of $\lambda$-calculus living in Cpo.

Let $\mathcal{A}, \mathcal{B}$ be information systems. If $f: A \rightarrow B$ is a function, we define $f^{*}: \mathcal{A}^{+} \rightarrow \mathcal{B}^{+}$and $f_{*}: \mathcal{B}^{+} \rightarrow \mathcal{A}^{+}$as follows:

- $f^{*}(x)=\overline{\{f(\alpha): \alpha \in x\}_{\mathcal{B}}}$, for every closed set $x$ of $\mathcal{A}$;

- $f_{*}(y)=\overline{\{\alpha: f(\alpha) \in y\}}_{\mathcal{A}}$, for every closed set $y$ of $\mathcal{B}$.

Lemma 4.1. The functions $f^{*}, f_{*}$ are Scott continuous.

The maps $f^{*}$ and $f_{*}$ are candidate to be a retraction, but we need more hypotheses.

The notions of backward morphism and forward morphism were introduced in [19].

Definition 4.2. A function $f: A \rightarrow B$ is a backward morphism (b-morphism, for short) from $\mathcal{A}=\left(A, \vdash_{\mathcal{A}}\right)$ to $\mathcal{B}=\left(B, \vdash_{\mathcal{B}}\right)$ if, for all $a \subseteq_{\mathrm{f}} A$ and $b \subseteq_{\mathrm{f}} B$, it satisfies:

(H1) $f(a) \vdash_{\mathcal{B}} f(b) \Rightarrow a \vdash_{\mathcal{A}} b$

The map $f$ is a forward morphism (f-morphism, for short) from $\mathcal{A}$ into $\mathcal{B}$ if it satisfies:

$(\mathrm{H} 2) a \vdash_{\mathcal{A}} b \Rightarrow f(a) \vdash_{\mathcal{B}} f(b)$ 
We leave to the reader the easy relativization of the notions of b-morphism and f-morphism to the case in which $f$ is a partial map.

Proposition 4.3. Let $f: \mathcal{A} \rightarrow \mathcal{B}$ be a b-morphism. Then $\left(f_{*}, f^{*}\right)$ is a retraction pair from $\mathcal{A}^{+}$into $\mathcal{B}^{+}$.

Proof. From (H1) it follows $f_{*} \circ f^{*}=\mathrm{id}_{\mathcal{A}^{+}}$.

Definition 4.4. A reflexive information system (a ris, for short) is a pair $\mathbf{A}=$ $\left(\mathcal{A}, \rightarrow_{\mathbf{A}}\right)$ where $\mathcal{A}$ is an information system and $\rightarrow_{\mathbf{A}}$ is a b-morphism from $\mathcal{A} \Rightarrow \mathcal{A}$ into $\mathcal{A}$.

The set of tokens of $\mathcal{A}$ is called the web of $\mathbf{A}$. In the following we will write $a \rightarrow \mathbf{A} b$ for $\rightarrow \mathbf{A}(a, b)$.

Corollary 4.5. Let $\mathbf{A}=\left(\mathcal{A}, \rightarrow_{\mathbf{A}}\right)$ be a ris, $F=$ fun $\circ\left(\rightarrow_{\mathbf{A}}\right)_{*}$ and $G=\left(\rightarrow_{\mathbf{A}}\right)^{*} \circ$ gph. Then $\mathbf{A}^{+}=\left(\mathcal{A}^{+}, F, G\right)$ is a reflexive object in the category ALat through the retraction pair $(F, G)$ from $\left[\mathcal{A}^{+} \rightarrow \mathcal{A}^{+}\right]$into $\mathcal{A}^{+}$.

The reflexive object $\mathbf{A}^{+}$will be called a ris-model.

\subsection{EXAMPLES OF RIS-MODELS}

In this subsection we explain how some known classes of models can be viewed as examples of ris-models.

\subsubsection{Krivine/Graph models as ris models}

A preordered set $(A, \leq)$, where $A$ is non-empty, defines an information system $\mathcal{A}=\left(A, \vdash_{\mathcal{A}}\right)$ as follows: $a \vdash_{\mathcal{A}} b$ iff $\forall \beta \in b . \exists \alpha \in a . \alpha \geq \beta$. In this context any function $\phi: \mathcal{P}_{\mathrm{f}}(A) \times \mathcal{P}_{\mathrm{f}}(A) \rightarrow A$ satisfying the following implication:

- if $\phi\left(a, a^{\prime}\right) \leq \phi\left(b, b^{\prime}\right)$, then $a \vdash_{\mathcal{A}} b$ and $b^{\prime} \vdash_{\mathcal{A}} a^{\prime}$

is a b-morphism, making $\mathbf{A}=(\mathcal{A}, \phi)$ a ris. Krivine models of $\lambda$-calculus [12, Section 5.6.2] arise from such ris's. Graph models [12, Section 5.5] arise from the restricted class of ris's in which the preorder $\leq$ is the equality.

\subsubsection{Filter models as ris-models}

The aim of the present section is to prove that every filter model living in Cpo is a ris-model. This is of course an explanation of the reason why our result concerning ris models does apply to the problem posed by Alessi and DezaniCiancaglini.

Let $\mathcal{S}=\left(S, \leq_{\mathcal{S}}, \wedge, \rightarrow, \omega\right)$ be an eats (see Section 2.2). As a matter of notation, if $a=\left\{\alpha_{1}, . ., \alpha_{n}\right\} \subseteq_{\mathrm{f}} \mathcal{S}$ we write $\wedge a$ as a shorthand for $\alpha_{1} \wedge \ldots \wedge \alpha_{n}$. We define an information system $\mathcal{A}_{\mathcal{S}}=\left(S, \vdash_{\mathcal{S}}\right)$ by setting $a \vdash_{\mathcal{S}} b\left(a, b \subseteq_{\mathrm{f}} S\right)$ iff $\wedge a \leq \mathcal{S} \wedge b($ a similar observation appears already in [22]).

In the exponential $\mathcal{A}_{\mathcal{S}} \Rightarrow \mathcal{A}_{\mathcal{S}}$ of $\mathcal{A}_{\mathcal{S}}$ we have $\left\{\left(a_{1}, b_{1}\right), \ldots,\left(a_{n}, b_{n}\right)\right\} \vdash_{\mathcal{A}_{\mathcal{S}} \Rightarrow \mathcal{A}_{\mathcal{S}}}$ $(c, d)$ iff either $\omega \leq \mathcal{S} \wedge d$ or there exists $J \subseteq\{1, \ldots, n\}, J \neq \emptyset$ such that $\wedge c \leq \mathcal{S}$ $\wedge\left(\cup_{j \in J} a_{j}\right)$ and $\wedge\left(\cup_{j \in J} b_{j}\right) \leq_{\mathcal{S}} \wedge d$. This condition results directly by instantiating the definition of the exponential $\mathcal{A}_{\mathcal{S}} \Rightarrow \mathcal{A}_{\mathcal{S}}$ given in Definition 3.4. 
In order to define a ris $\left(\mathcal{A}_{\mathcal{S}}, \phi_{\mathcal{S}}\right)$ we now define $\phi_{\mathcal{S}}: \mathcal{P}_{\mathrm{f}}(S) \times \mathcal{P}_{\mathrm{f}}(S) \rightarrow S$ by setting $\phi_{\mathcal{S}}(a, b)=\wedge a \rightarrow \wedge b$.

Proposition 4.6. Let $\mathcal{S}$ be an eats satisfying condition (C3) as in Theorem 2.5. Then

(i) $\mathbf{A}_{\mathcal{S}}=\left(\mathcal{A}_{\mathcal{S}}, \phi_{\mathcal{S}}\right)$ is a ris;

(ii) The ris-model $\mathbf{A}_{\mathcal{S}}^{+}$coincides with the filter model $\left(\mathcal{F}_{\mathcal{S}}, F, G\right)$ determined by the eats $\mathcal{S}$.

Proof.

(i) The function $\phi_{\mathcal{S}}$ is a b-morphism, i.e. , it satisfies the implication

$\left\{\phi_{\mathcal{S}}\left(a_{1}, b_{1}\right), \ldots, \phi_{\mathcal{S}}\left(a_{n}, b_{n}\right)\right\} \vdash_{\mathcal{A}_{\mathcal{S}}} \phi_{\mathcal{S}}(a, b) \Rightarrow\left\{\left(a_{1}, b_{1}\right), \ldots,\left(a_{n}, b_{n}\right)\right\} \vdash_{\mathcal{A}_{\mathcal{S}} \Rightarrow \mathcal{A}_{\mathcal{S}}}(a, b)$

if, and only if, $\mathcal{S}$ satisfies condition (C3). Thus $\mathbf{A}_{\mathcal{S}}$ is a ris.

(ii) Recall by Corollary 4.5 that the maps $F^{\prime}$ and $G^{\prime}$, defined by $F^{\prime}=$ fun $\circ$ $\left(\phi_{\mathcal{S}}\right)_{*}$ and $G^{\prime}=\left(\phi_{\mathcal{S}}\right)^{*} \circ$ gph, make $\mathbf{A}_{\mathcal{S}}^{+}$a ris-model. Let $X \subseteq S$ be a closed set of the information system $\mathcal{A}_{\mathcal{S}}$. Then $X$ is upward closed w.r.t. $\leq$ and closed under $\wedge$, so that it is a filter; conversely, every filter is a closed subset of $S$. Therefore $\mathcal{A}_{\mathcal{S}}^{+}=\mathcal{F}_{\mathcal{S}}$. Moreover the closure operator $\overline{(\cdot)_{\mathcal{S}}}$ coincides with $\uparrow(\cdot)$. Now recall the definitions of $F: \mathcal{F}_{\mathcal{S}} \rightarrow\left[\mathcal{F}_{\mathcal{S}} \rightarrow \mathcal{F}_{\mathcal{S}}\right]$ and $G:\left[\mathcal{F}_{\mathcal{S}} \rightarrow \mathcal{F}_{\mathcal{S}}\right] \rightarrow \mathcal{F}_{\mathcal{S}}$ from Section 2.2, the definitions of gph and fun from the proof of Proposition 3.5; look at Section 4 in order to work out the definitions of $\left(\phi_{\mathcal{S}}\right)^{*}$ and $\left(\phi_{\mathcal{S}}\right)_{*}$.

$$
\begin{aligned}
F^{\prime}(X)(Y) & =\bigcup\left\{b \subseteq_{\mathrm{f}} S: \exists a \subseteq_{\mathrm{f}} Y .(a, b) \in \uparrow\{(a, b): \wedge a \rightarrow \wedge b \in X\}\right\} \\
& =\{\beta \in S: \exists \alpha \in Y . \alpha \rightarrow \beta \in X\} \\
& =F(X)(Y) \\
G^{\prime}(f) & =\uparrow\left\{\wedge a \rightarrow \wedge b:(a, b) \in\left\{\left(a^{\prime}, b^{\prime}\right): b^{\prime} \subseteq_{\mathrm{f}} f\left(\uparrow a^{\prime}\right)\right\}\right\} \\
& =\uparrow\{\alpha \rightarrow \beta \in S: \beta \in f(\uparrow \alpha)\} \\
& =G(f)
\end{aligned}
$$

This shows that the filter $\lambda$-model $\mathcal{F}_{\mathcal{S}}$ coincides with $\mathbf{A}_{\mathcal{S}}^{+}$, and hence it is a ris-model too.

\subsection{The interpretation of $\lambda$-TERMS In A RIS-MODEL}

In this section we make explicit the definition of interpretation of a $\lambda$-term as a closed subset of a ris, instantiating the more abstract definition of interpretation in a $\lambda$-model (see Section 2.1.1).

Let $\mathbf{A}=\left(\mathcal{A}, \rightarrow_{\mathbf{A}}\right)$ be a ris and $E_{n v_{\mathbf{A}}}$ be the set of all finite environments, that is, functions from $\operatorname{Var}$ into $\mathcal{P}_{\mathrm{f}}(A)$. The interpretation $\llbracket M \rrbracket_{\rho}^{\mathbf{A}}$ of a $\lambda$-term $M$ in a 
finite environment $\rho$ can be also described by a system of judgements of the form $\rho \triangleright_{\mathbf{A}} M: a$ (where $a \subseteq_{\mathrm{f}} A$ ) whose intended meaning will be $a \subseteq_{\mathrm{f}} \llbracket M \rrbracket_{\rho}^{\mathbf{A}}$. As usual, we write $\rho \triangleright_{\mathbf{A}} M: \alpha$ for $\rho \triangleright_{\mathbf{A}} M:\{\alpha\}$.

Two environments $\rho$ and $\sigma$ are called $\mathcal{A}$-equivalent if $\left\{\alpha: \rho(y) \vdash_{\mathcal{A}} \alpha\right\}=\{\alpha$ : $\left.\sigma(y) \vdash_{\mathcal{A}} \alpha\right\}$ for all variables $y$. We can simultaneously define the interpretation $\llbracket M \rrbracket_{\rho}^{\mathbf{A}}$ of a $\lambda$-term $M$ and show that this interpretation is independent of the choice of $\mathcal{A}$-equivalent environments:

$$
\begin{aligned}
\llbracket y \rrbracket_{\rho}^{\mathbf{A}} & =\left\{\alpha: \rho(y) \vdash_{\mathcal{A}} \alpha\right\} \\
\llbracket \lambda y \cdot M \rrbracket_{\rho}^{\mathbf{A}}= & G\left(\bar{a}_{\mathcal{A}} \mapsto \llbracket M \rrbracket_{\rho[y:=a]}^{\mathbf{A}}\right), \text { where } G=\left(\rightarrow_{\mathbf{A}}\right)^{*} \circ \text { gph } \\
= & \left\{\alpha: \exists d \subseteq_{\mathrm{f}} A \cdot d \vdash_{\mathcal{A}} \alpha \text { and } d \subseteq_{\mathrm{f}}\left\{a \rightarrow_{\mathbf{A}} b: b \subseteq_{\mathrm{f}} \llbracket M \rrbracket_{\rho[y:=a]}^{\mathbf{A}}\right\}\right\} \\
\llbracket M N \rrbracket_{\rho}^{\mathbf{A}}= & F\left(\llbracket M \rrbracket_{\rho}^{\mathbf{A}}\right)\left(\llbracket N \rrbracket_{\rho}^{\mathbf{A}}\right), \text { where } F=\text { fun } \circ\left(\rightarrow_{\mathbf{A}}\right)_{*} \\
= & \bigcup\left\{b: \exists a \subseteq_{\mathrm{f}} \llbracket N \rrbracket_{\rho}^{\mathbf{A}} \cdot \exists x \subseteq A \cdot x \vdash_{\mathcal{A} \Rightarrow_{\mathcal{A}}(a, b) \text { and }}\right. \\
& \left.x \subseteq_{\mathrm{f}}\left\{(c, d): c \rightarrow_{\mathbf{A}} d \in \llbracket M \rrbracket_{\rho}^{\mathbf{A}}\right\}\right\} \\
= & \bigcup\left\{b: \exists a \subseteq_{\mathrm{f}} \llbracket N \rrbracket_{\rho}^{\mathbf{A}} \cdot \exists e \subseteq_{\mathrm{f}} \llbracket M \rrbracket_{\rho}^{\mathbf{A}} \cdot e=\left\{c_{1} \rightarrow_{\mathbf{A}} d_{1}, \ldots, c_{n} \rightarrow_{\mathbf{A}} d_{n}\right\},\right. \\
& \left.a \vdash_{\mathcal{A}} \cup c_{i} \text { and } \cup d_{i} \vdash_{\mathcal{A}} b\right\}
\end{aligned}
$$

We advise the reader to distinguish the entailment relation $\vdash_{\mathcal{A}}$ of the information system $\mathcal{A}$ and the entailment relation $\triangleright_{\mathbf{A}}$ associated with the ris $\mathbf{A}$ we will now define.

The following are the deduction rules:

$$
\begin{array}{cc}
{ } M: \emptyset }\left[r_{0}\right] } & \frac{\rho \triangleright_{\mathbf{A}} y: \rho(y)}{\left.\rho r_{1}\right]} \\
\frac{\rho \triangleright_{\mathbf{A}} M: a}{\rho \triangleright_{\mathbf{A}} M: b} \quad a \cup b \vdash_{\mathcal{A}} c \\
\rho \triangleright_{\mathbf{A}} M: c & \frac{\rho[y:=a] \triangleright_{\mathbf{A}} M: b}{\rho \triangleright_{\mathbf{A}} \lambda y \cdot M: a \rightarrow_{\mathbf{A}} b}\left[r_{3}\right] \\
\frac{\rho \triangleright_{\mathbf{A}} M: a \rightarrow_{\mathbf{A}} b \quad \rho \triangleright_{\mathbf{A}} N: a}{\rho \triangleright_{\mathbf{A}} M N: b}\left[r_{4}\right] &
\end{array}
$$

Notice that by rule $\left(r_{2}\right)$ we have that $\rho \triangleright_{\mathbf{A}} M: a$ iff $\rho \triangleright_{\mathbf{A}} M: \alpha$ for all $\alpha \in a$.

Proposition 4.7. Let $\mathbf{A}=\left(\mathcal{A}, \rightarrow_{\mathbf{A}}\right)$ be a ris and $\rho$ be a finite environment. Then $b \subseteq_{\mathrm{f}} \llbracket M \rrbracket_{\rho}^{\mathbf{A}}$ iff $\rho \triangleright_{\mathbf{A}} M: b$.

Proof. We proceed by induction on the structure of terms. The base of the induction is obvious.

$(M \equiv \lambda y . P):$ If $b \subseteq_{\mathrm{f}} \llbracket \lambda y . P \rrbracket_{\rho}^{\mathbf{A}}$ then there exist $a_{i} \rightarrow_{\mathbf{A}} b_{i}(i=1, \ldots, n)$ such that $b_{i} \subseteq_{\mathrm{f}} \llbracket P \rrbracket_{\rho\left[y:=a_{i}\right]}^{\mathbf{A}}$ and $\left\{a_{i} \rightarrow_{\mathbf{A}} b_{i}: 1 \leq i \leq n\right\} \vdash_{\mathcal{A}} b$. By induction hypothesis $\rho\left[y:=a_{i}\right] \triangleright_{\mathbf{A}} P: b_{i}$, so that by applying rule $\left(r_{3}\right)$ and $\left(r_{2}\right)$ we get first $\rho \triangleright_{\mathbf{A}} \lambda y \cdot P$ : $a_{i} \rightarrow_{\mathbf{A}} b_{i}$ and then $\rho \triangleright_{\mathbf{A}} \lambda y . P:\left\{a_{i} \rightarrow_{\mathbf{A}} b_{i}: 1 \leq i \leq n\right\}$. Finally, an application of $\left(r_{2}\right)$ to this last entailment and to $\left\{a_{i} \rightarrow_{\mathbf{A}} b_{i}: 1 \leq i \leq n\right\} \vdash_{\mathcal{A}} b$ provides the conclusion. We now show the opposite direction. If we have $\rho \triangleright_{\mathbf{A}} \lambda y \cdot P: b$ by applying $\left(r_{2}\right)$ the conclusion easily follows. If we have applied $\left(r_{3}\right)$ then we have 
that $b=\left\{c \rightarrow_{\mathbf{A}} d\right\}$ and $\rho[y:=c] \triangleright_{\mathbf{A}} P: d$. By induction hypothesis $d \subseteq_{\mathrm{f}} \llbracket P \rrbracket_{\rho[y:=c]}^{\mathbf{A}}$, so that by definition of interpretation $c \rightarrow_{\mathbf{A}} d \in \llbracket P \rrbracket_{\rho}^{\mathbf{A}}$.

$(M \equiv P Q):$ If $b \subseteq_{\mathrm{f}} \llbracket P Q \rrbracket_{\rho}^{\mathbf{A}}$ then there exist $a \subseteq \llbracket Q \rrbracket_{\rho}^{\mathbf{A}}$ and $c_{i}, d_{i}(i \leq n)$ such that $c_{i} \rightarrow_{\mathbf{A}} d_{i} \in \llbracket P \rrbracket_{\rho}^{\mathbf{A}}$ with $a \vdash_{\mathcal{A}} c_{i}$ for all $i$ and $d_{1} \cup \cdots \cup d_{n} \vdash_{\mathcal{A}} b$. From the fact that $\llbracket Q \rrbracket_{\rho}^{\mathbf{A}}$ is closed, $a \subseteq \llbracket Q \rrbracket_{\rho}^{\mathbf{A}}$ and $a \vdash_{\mathcal{A}} c_{i}$ it follows that $c_{i} \subseteq \llbracket Q \rrbracket_{\rho}^{\mathbf{A}}$. By induction hypothesis we have that $\rho \triangleright_{\mathbf{A}} Q: c_{i}$ and $\rho \triangleright_{\mathbf{A}} P: c_{i} \rightarrow_{\mathbf{A}} d_{i}$, so that by rule $\left(r_{4}\right)$ $\rho \triangleright_{\mathbf{A}} P Q: d_{i}$ for all $i=1, \ldots, n$. Then $\rho \triangleright_{\mathbf{A}} P Q: d_{1} \cup \cdots \cup d_{n}$ by rule $\left(r_{2}\right)$. Finally, by applying rule $\left(r_{2}\right)$ to $d_{1} \cup \cdots \cup d_{n} \vdash_{\mathcal{A}} b$ and to $\rho \triangleright_{\mathbf{A}} P Q: d_{1} \cup \cdots \cup d_{n}$ we get $\rho \triangleright_{\mathbf{A}} P Q: b$. The opposite direction is easy.

\section{Completion method}

When dealing with constructions of webbed models with special purposes, it is indeed very useful to have canonical procedures for completing finite pieces of web. This idea dates back to Longo [35] and has been further developed by Kerth [30]. This method is useful for building models satisfying prescribed constraints, such as domain equations and inequations, and it is particularly convenient for dealing with the equational theories of webbed models. The completion method presented in this section has been fruitfully applied in [19] to show that the least extensional $\lambda$-theory $\lambda \beta \eta$ cannot be the theory of a reflexive Scott domain in the category Cpo.

\subsection{PARTIAL RIS'S}

A partial reflexive information system (a partial ris, for short) is a pair $\mathbf{A}=$ $\left(\mathcal{A}, \rightarrow_{\mathbf{A}}\right)$, where $\mathcal{A}$ is an information system and $\rightarrow_{\mathbf{A}}:(\mathcal{A} \Rightarrow \mathcal{A}) \rightarrow \mathcal{A}$ is a partial b-morphism. For the rest of the paper is very important to notice that $\lambda$-terms can be interpreted as subsets of a partial ris $\mathbf{A}$ by using the above deduction rules $\left(r_{0}\right)-\left(r_{1}\right)-\left(r_{2}\right)-\left(r_{4}\right)$ and

$$
\frac{\rho[y:=a] \triangleright_{\mathbf{A}} M: b \quad(a, b) \in \operatorname{dom}\left(\rightarrow_{\mathbf{A}}\right)}{\rho \triangleright_{\mathbf{A}} \lambda y \cdot M: a \rightarrow_{\mathbf{A}} b}\left[r_{3}^{\prime}\right]
$$

A finite ris is a partial ris with a finite number of tokens.

Definition 5.1. Let $\mathbf{B}=\left(\mathcal{B}, \rightarrow_{\mathbf{B}}\right)$ be a partial ris and $\mathbf{C}=\left(\mathcal{C}, \rightarrow_{\mathbf{C}}\right)$ be a ris. We say that $h: \mathbf{B} \rightarrow \mathbf{C}$ is a ris $f$-morphism if $h: \mathcal{B} \rightarrow \mathcal{C}$ is an f-morphism of information systems and the following further condition holds:

$(\mathrm{H} 3) h\left(a \rightarrow_{\mathbf{B}} b\right)=h(a) \rightarrow_{\mathbf{C}} h(b)$, for all $(a, b) \in \operatorname{dom}\left(\rightarrow_{\mathbf{B}}\right)$.

Lemma 5.2. If $h$ is a ris $f$-morphism from a partial ris $\mathbf{B}=\left(\mathcal{B}, \rightarrow_{\mathbf{B}}\right)$ into a ris $\mathbf{C}=(\mathcal{C}, \rightarrow \mathbf{C})$, then we have:

$$
\rho \triangleright_{\mathbf{B}} M: a \Rightarrow h(\rho) \triangleright_{\mathbf{C}} M: h(a) .
$$


Proof. The proof is by induction on the length of the proof of $\rho \triangleright_{\mathbf{B}} M: a$.

Let $\pi$ be a proof $\rho \triangleright_{\mathbf{B}} y: \rho(y)$ consisting of an application of rule $\left(r_{1}\right)$. Then $h(\rho) \triangleright_{\mathbf{C}} y: h(\rho(y))$ is trivially true.

Let $\pi$ be a proof of $\rho \triangleright_{\mathbf{B}} M: \emptyset$ consisting of an application of rule $\left(r_{0}\right)$. Then $h(\rho) \triangleright_{\mathbf{B}} M: h(\emptyset)=\emptyset$.

Let $\pi$ be a proof of $\rho \triangleright_{\mathbf{B}} M: c$ consisting of an application of rule $\left(r_{2}\right)$ to a proof $\pi_{1}$ of $\rho \triangleright_{\mathbf{B}} M: a$ and a proof $\pi_{2}$ of $\rho \triangleright_{\mathbf{B}} M: b$, where $a \cup b \vdash_{\mathcal{B}} c$. Then

$$
\frac{h(\rho) \triangleright_{\mathbf{C}} M: h(a) \quad h(\rho) \triangleright_{\mathbf{C}} M: h(b) \quad h(a \cup b)=h(a) \cup h(b) \vdash_{\mathcal{C}} h(c)}{\rho \triangleright_{\mathbf{C}} M: h(c)}
$$

follows from the hypothesis that $h$ is an f-morphism from $\mathcal{B}$ into $\mathcal{C}$, so that $a \cup b \vdash_{\mathcal{B}} c$ implies $h(a \cup b) \vdash_{\mathcal{C}} h(c)$.

Let $\pi$ be a proof of $\rho \triangleright_{\mathbf{B}} \lambda y . M: a \rightarrow_{\mathbf{B}} b$ consisting of an application of rule $\left(r_{3}^{\prime}\right)$ to a proof $\pi_{1}$ of $\rho[y:=a] \triangleright_{\mathbf{A}} M: b$, assuming $(a, b) \in \operatorname{dom}\left(\rightarrow_{\mathbf{B}}\right)$. Then

$$
\frac{h(\rho[y:=a])=h(\rho)[y:=h(a)] \triangleright_{\mathbf{C}} M: h(b)}{h(\rho) \triangleright_{\mathbf{C}} \lambda y \cdot M: h(a) \rightarrow_{\mathbf{C}} h(b)}
$$

Let $\pi$ be a proof of $\rho \triangleright_{\mathbf{B}} M N: b$ whose conclusion is obtained by an application of rule $\left(r_{4}\right)$, to a proof $\pi_{1}$ of $\rho \triangleright_{\mathbf{B}} M: a \rightarrow_{\mathbf{B}} b$ and a proof $\pi_{2}$ of $\rho \triangleright_{\mathbf{B}} N: a$. Then

$$
\frac{h(\rho) \triangleright_{\mathbf{C}} M: h\left(a \rightarrow_{\mathbf{B}} b\right)=h(a) \rightarrow_{\mathbf{C}} h(b) \quad h(\rho) \triangleright_{\mathbf{C}} N: h(a)}{h(\rho) \triangleright_{\mathbf{C}} M N: h(b)}
$$

follows from the hypothesis that $h\left(a \rightarrow_{\mathbf{B}} b\right)=h(a) \rightarrow_{\mathbf{C}} h(b)$.

Definition 5.3. A partial ris $\mathbf{A}=\left(\mathcal{A}, \rightarrow_{\mathbf{A}}\right)$ is a subsystem of a ris $\mathbf{B}=\left(\mathcal{B}, \rightarrow_{\mathbf{B}}\right)$ if the following conditions hold:

- $A \subseteq B$ and $\vdash_{\mathcal{A}}=\vdash_{\mathcal{B}} \cap\left(\mathcal{P}_{\mathrm{f}}(A) \times \mathcal{P}_{\mathrm{f}}(A)\right)$.

- $\rightarrow_{\mathbf{A}}=\rightarrow_{\mathbf{B}} \cap\left(\left(\mathcal{P}_{\mathrm{f}}(A) \times \mathcal{P}_{\mathrm{f}}(A)\right) \times A\right)$.

Notice that the subsystem $\mathbf{A}$ of $\mathbf{B}$ is univocally characterized by the subset $A$ of $B$. In other words, given $A \subseteq B, \vdash_{\mathcal{A}}$ and $\rightarrow_{\mathbf{A}}$ are univocally characterized by the conditions expressed in the above definition.

\subsection{The COMPLETion}

Starting from a partial ris $\mathbf{A}=(\mathcal{A}, \rightarrow \mathbf{A})$, it is possible to obtain by "completion" a (total) ris $\mathbf{A}_{\omega}=\left(\mathcal{A}_{\omega}, \rightarrow_{\omega}\right)$ such that $\rightarrow_{\omega}$ and $\mathcal{A}_{\omega}$ extend respectively $\rightarrow_{\mathbf{A}}$ and $\mathcal{A}$.

The canonical completion $\mathbf{A}_{\omega}=\left(\mathcal{A}_{\omega}, \rightarrow \omega\right)$ of a partial ris $\mathbf{A}=\left(\mathcal{A}, \rightarrow_{\mathbf{A}}\right)$ (where w.l.o.g. we assume $A$ does not contain pairs) is defined as follows:

$$
A_{0}=A ; \quad A_{n+1}=A_{n} \cup\left(\left(\mathcal{P}_{\mathrm{f}}\left(A_{n}\right) \times \mathcal{P}_{\mathrm{f}}\left(A_{n}\right)\right)-\operatorname{dom}\left(\rightarrow_{\mathbf{A}}\right)\right)
$$




$$
\begin{aligned}
& \text { - } A_{\omega}=\cup_{n} A_{n} \\
& \text { - } a \vdash_{\omega} b \text { iff }(a \cap A) \vdash_{\mathcal{A}}(b \cap A) \text { and } b \cap\left(A_{\omega}-A\right) \subseteq a \cap\left(A_{\omega}-A\right) \\
& \text { - } \mathcal{A}_{\omega}=\left(A_{\omega}, \vdash_{\omega}\right) \\
& \text { - } a \rightarrow_{\omega} b= \begin{cases}a \rightarrow_{\mathbf{A}} b & \text { if }(a, b) \in \operatorname{dom}\left(\rightarrow_{\mathbf{A}}\right) \\
(a, b) & \text { otherwise }\end{cases}
\end{aligned}
$$

Lemma 5.4. The canonical completion $\mathbf{A}_{\omega}$ of a partial ris $\mathbf{A}$ is a ris.

Proof. First we observe that $\mathcal{A}_{\omega}$ is an information system, since the relation $\vdash_{\omega}$ is an entailment. Moreover the map $\rightarrow_{\omega}$ is a total b-morphism from $\mathcal{A}_{\omega} \Rightarrow \mathcal{A}_{\omega}$ to $\mathcal{A}_{\omega}$.

Lemma 5.5. Let $\mathbf{A}=\left(\mathcal{A}, \rightarrow_{\mathbf{A}}\right)$ be a partial ris, which is a subsystem of a ris $\mathbf{B}=\left(\mathcal{B}, \rightarrow_{\mathbf{B}}\right)$. Then there exists a ris f-morphism $g_{\omega}$ from the canonical completion $\mathbf{A}_{\omega}$ of $\mathbf{A}$ into $\mathbf{B}$.

Proof. Recall that $A_{\omega}=\cup_{n \in \mathbb{N}} A_{n}$, where $A_{0}=A$ and $A_{n+1}-A$ is a set of pairs. We define $g_{\omega}$ by induction as follows:

$$
g_{\omega}(\alpha)= \begin{cases}\alpha, & \text { if } \alpha \in A_{0}=A \\ g_{\omega}(a) \rightarrow_{\mathbf{B}} g_{\omega}(b), & \text { if } \alpha \equiv(a, b) \in A_{n+1}-A_{n} .\end{cases}
$$

Condition (H2) of Definition 4.2 is straightforward to verify, since $\vdash_{\omega}$ coincides with $\supseteq_{\mathrm{f}}$ for elements of $A_{\omega}-A$.

We now show condition (H3) of Definition 5.1: If $(a, b) \in \operatorname{dom}\left(\rightarrow_{\mathbf{A}}\right)$ then $g_{\omega}\left(a \rightarrow_{\omega} b\right)=g_{\omega}\left(a \rightarrow_{\mathbf{A}} b\right)=a \rightarrow_{\mathbf{A}} b=a \rightarrow_{\mathbf{B}} b=g_{\omega}(a) \rightarrow_{\mathbf{B}} g_{\omega}(b)$, because $g_{\omega}$ is the identity restricted to the elements of $A$ and $\rightarrow_{\mathbf{A}}=\rightarrow_{\mathbf{B}}$ over the elements of $\operatorname{dom}(\rightarrow \mathbf{A})$.

If $(a, b) \notin \operatorname{dom}\left(\rightarrow_{\mathbf{A}}\right)$ then $g_{\omega}\left(a \rightarrow_{\omega} b\right)=g_{\omega}(a, b)=g_{\omega}(a) \rightarrow_{\mathbf{B}} g_{\omega}(b)$ by definition of $g_{\omega}$ over the pairs.

Recall that an inequality $M \sqsubseteq N$ fails in a ris $\mathbf{B}$ if there exists a finite $B$ environment $\rho$ such that $\llbracket M \rrbracket_{\rho}^{\mathrm{B}} \nsubseteq \llbracket N \rrbracket_{\rho}^{\mathrm{B}}$.

The following is the main theorem of the section.

Theorem 5.6. Let $\mathbf{B}=\left(\mathcal{B}, \rightarrow_{\mathbf{B}}\right)$ be a ris and $M \sqsubseteq N$ be an inequality which fails in $\mathbf{B}$. Then there exists a finite ris $\mathbf{A}$ such that $M \sqsubseteq N$ fails in the canonical completion $\mathbf{A}_{\omega}$ of $\mathbf{A}$.

Proof. The proof is divided into two parts.

In the first part we illustrate a way to construct, given a proof $\pi$ of $\rho \triangleright_{\mathbf{B}} M: \alpha$ a subset $A_{\pi} \subseteq B$ of tokens containing $\alpha$. Intuitively, this will induce a finite subsystem of $\mathbf{B}$ whose canonical completion satisfies: $\alpha$ is in the interpretation of $M$. The construction is by induction on the height of proofs.

Let $\pi$ be a proof of $\rho \triangleright_{\mathbf{B}} y: \rho(y)$ consisting of an application of rule $\left(r_{1}\right)$. Then we define $A_{\pi}=\rho(y)$.

Let $\pi$ be a proof of $\rho \triangleright_{\mathbf{B}} M: \emptyset$ consisting of an application of rule $\left(r_{0}\right)$. In this case define $A_{\pi}=\emptyset$. 
Let $\pi$ be a proof of $\rho \triangleright_{\mathbf{B}} M: c$ consisting of an application of rule $\left(r_{2}\right)$ to a proof $\pi_{1}$ of $\rho \triangleright_{\mathbf{B}} M: a$ and a proof $\pi_{2}$ of $\rho \triangleright_{\mathbf{B}} M: b$, where $a \cup b \vdash_{\mathcal{B}} c$. In this case, assume that we have already constructed $A_{\pi_{1}}$ and $A_{\pi_{2}}$, define $A_{\pi}=A_{\pi_{1}} \cup A_{\pi_{2}} \cup c$. Let $\pi$ be a proof of $\rho \triangleright_{\mathbf{B}} \lambda y \cdot M: a \rightarrow_{\mathbf{B}} b$ consisting of an application of rule $\left(r_{3}\right)$ to a proof $\pi_{1}$ of $\rho[y:=a] \triangleright_{\mathbf{A}} M: b$. In this case define $A_{\pi}=A_{\pi_{1}} \cup\left\{a \rightarrow_{\mathbf{B}} b\right\}$.

Let $\pi$ be a proof of $\rho \triangleright_{\mathbf{B}} M N: b$ whose conclusion is obtained by an application of rule $\left(r_{4}\right)$, to a proof $\pi_{1}$ of $\rho \triangleright_{\mathbf{B}} M: a \rightarrow_{\mathbf{B}} b$ and a proof $\pi_{2}$ of $\rho \triangleright_{\mathbf{B}} N: a$. Assume that we have already defined $A_{\pi_{1}}, A_{\pi_{2}} \subseteq B$. Then we define $A_{\pi}=A_{\pi_{1}} \cup A_{\pi_{2}} \cup b$, because $a \rightarrow_{\mathbf{B}} b \in A_{\pi_{1}}$ and $a \subseteq A_{\pi_{2}}$ but it may happen that $b \nsubseteq_{\mathrm{f}} A_{\pi_{1}} \cup A_{\pi_{2}}$.

In the second part of the proof we use the above construction in order to exhibit a finite ris $\mathbf{A}$ such that $M \sqsubseteq N$ fails in the canonical completion $\mathbf{A}_{\omega}$ of $\mathbf{A}$.

Let $\alpha \in \llbracket M \rrbracket_{\rho}^{\mathbf{B}}-\llbracket N \rrbracket_{\rho}^{\mathbf{B}}$ for some finite $B$-environment $\rho$. By Proposition 4.7 we have that $\alpha \in \llbracket M \rrbracket_{\rho}^{\mathbf{B}}$ iff there is a proof $\pi$ of $\rho \triangleright_{\mathbf{B}} M: \alpha$. Let $A=A_{\pi}$ be the subset of $B$ resulting from the construction described in the first part. Recall from Definition 5.3 that $\vdash_{\mathcal{A}}$ and $\rightarrow_{\mathbf{A}}$ are univocally determined by the set $A$.

We let $\rho_{A}$ be the $A$-environment defined by $\rho_{A}(x)=\rho(x) \cap A$ for every variable $x$. It is evident that the proof $\pi$ of $\rho \triangleright_{\mathbf{B}} M: \alpha$ can be relativized to the finite ris $\mathbf{A}$ (so that it can be relativized to the canonical completion $\mathbf{A}_{\omega}$ of $\mathbf{A}$ ) by obtaining a proof $\pi_{A}$ of $\rho_{A} \triangleright_{\mathbf{A}} M: \alpha$. It follows that $\alpha \in \llbracket M \rrbracket_{\rho_{A}}^{\mathbf{A}_{\omega}}$. We now conclude the proof of the theorem by showing that $\alpha \notin \llbracket N \rrbracket_{\rho_{A}}^{\mathbf{A}_{\omega}}$. Assume, by way of contradiction, that

$\alpha \in \llbracket N \rrbracket_{\rho_{A}}^{\mathbf{A}_{\omega}}$. From Lemma 5.5 there exists a ris f-morphism $g_{\omega}: \mathbf{A}_{\omega} \rightarrow \mathbf{B}$. Since $\alpha \in A, \rho_{A}(x) \subseteq A$ for every $x, g_{\omega}$ is the identity restricted to $A$ and $g_{\omega}\left(\rho_{A}\right)=\rho_{A}$, then by Lemma 5.2 we have that $g_{\omega}(\alpha)=\alpha \in \llbracket N \rrbracket_{\rho_{A}}^{\mathrm{B}} \subseteq \llbracket N \rrbracket_{\rho}^{\mathrm{B}}$. This contradicts the original hypothesis.

\section{EFFECTIVENESS}

In this section we introduce the notion of an effective ris. The interested reader may refer to [19] for a more general theory of effectiveness.

Definition 6.1. We say that a ris $\mathbf{A}=(\mathcal{A}, \rightarrow \mathbf{A})$ is effective if there exists a bijective map $\sigma$ from $A$ onto the set $\mathbb{N}$ of natural numbers such that, after encoding, the entailment relation $\vdash_{\mathcal{A}}$ is decidable and the function $\rightarrow_{\mathbf{A}}: \mathcal{P}_{\mathrm{f}}(A) \times \mathcal{P}_{\mathrm{f}}(A) \rightarrow A$ is computable with a decidable range.

Theorem 6.2. The canonical completion of a finite ris is effective.

Proof. Let $\mathbf{A}=\left(\mathcal{A}, \rightarrow_{\mathbf{A}}\right)$ be a finite ris. By construction there exists a bijective correspondence between $A_{\omega}$ and the set $\mathbb{N}$ of natural numbers. The relation $\vdash_{\omega}$ is trivially decidable because $a \vdash_{\omega} \alpha$ iff either $\alpha \in a$ or $a \cap A \vdash_{\mathcal{A}} \alpha$ and $A$ is a finite set. Moreover, $\rightarrow \omega$ is the identity map in the cofinite set $\mathcal{P}_{\mathrm{f}}\left(A_{\omega}\right) \times \mathcal{P}_{\mathrm{f}}\left(A_{\omega}\right)-\operatorname{dom}\left(\rightarrow_{\mathbf{A}}\right)$ and $\rightarrow_{\mathbf{A}}$ is a finite function.

Lemma 6.3. Let $\mathbf{A}_{\omega}$ be the canonical completion of a finite ris $\mathbf{A}$. Then, after encoding, we have:

(i) $\llbracket N \rrbracket^{\mathbf{A}_{\omega}}$ is r.e. , for every $N \in \Lambda^{o}$ 
(ii) $\llbracket \lambda x . x \rrbracket^{\mathbf{A}_{\omega}}$ is decidable.

(iii) $\left\{M \in \Lambda^{o}: \llbracket M \rrbracket^{\mathbf{A}_{\omega}} \subseteq \llbracket \lambda x . x \rrbracket^{\mathbf{A}_{\omega}}\right\}$ is a co-r.e. set of $\lambda$-terms.

Proof. (i) Since $\vdash_{\omega}$ is decidable, the deduction rules $\left(r_{0}\right)-\left(r_{4}\right)$ in Section 4.2 are effective. Then the interpretation of a $\lambda$-term is r.e.

(ii) The set $\llbracket \lambda x . x \rrbracket^{\mathbf{A}_{\omega}}$ is the closure in $\mathcal{A}_{\omega}$ of the set $\left\{a \rightarrow_{\omega} b: b \subseteq_{\mathrm{f}} \overline{(a)}_{\mathcal{A}_{\omega}}\right\}$. Formally, being $\emptyset$ the empty environment, we have:

$$
\begin{aligned}
\llbracket \lambda x . x \rrbracket^{\mathbf{A}_{\omega}=} & \left\{\alpha: \exists d \subseteq_{\mathrm{f}} X . d \vdash_{\omega} \alpha\right\}, \quad \text { (see Section 4.2) } \\
& \text { where } X=\left\{a \rightarrow \rightarrow_{\omega} b: b \subseteq_{\mathrm{f}} \llbracket x \rrbracket_{\emptyset\left[x:=\overline{(a)}_{\left.\mathcal{A}_{\omega}\right]}\right\}}^{\mathbf{A}_{\omega}}\right\} \\
= & \left\{\alpha: \exists a_{i}, b_{i} \subseteq_{\mathrm{f}} A_{\omega} \cdot\left\{a_{1} \rightarrow_{\omega} b_{1}, \ldots, a_{n} \rightarrow_{\omega} b_{n}\right\} \vdash_{\omega} \alpha\right\}, \\
& \text { with } a_{i} \vdash_{\omega} b_{i} \\
= & \left\{(a, b) \in A_{\omega}-A: a \vdash_{\omega} b\right\} \cup\left\{\alpha \in A: \exists a_{i}, b_{i} \subseteq_{\mathrm{f}} A .\right. \\
& \left.a_{i} \vdash_{\mathcal{A}} b_{i} \text { and }\left\{a_{1} \rightarrow_{\mathbf{A}} b_{1}, \ldots, a_{n} \rightarrow_{\mathbf{A}} b_{n}\right\} \vdash_{\mathcal{A}} \alpha\right\}
\end{aligned}
$$

We outline an algorithm to decide the set $\llbracket \lambda x . x \rrbracket^{\mathbf{A}_{\omega}}$ :

- Given $\alpha \in A_{\omega}$ decide whether $\alpha \in A$ or $\alpha=(a, b) \in A_{\omega}-A$;

$(\alpha \in A)$ Search the finite set $A$ in order to find $a_{1} \rightarrow_{\mathbf{A}} b_{1}, \ldots, a_{n} \rightarrow_{\mathbf{A}} b_{n}$, which together entail $\alpha$ in $\mathcal{A}$. Output "no" iff this search fails.

$(\alpha \notin A)$ Output "yes" iff $a \vdash_{\omega} b$ (recall that $\vdash_{\omega}$ is decidable).

(iii) Since $\llbracket \lambda x . x \rrbracket^{\mathbf{A}_{\omega}}$ is a decidable closed subset of $A_{\omega}$, then $\left\{M \in \Lambda^{o}\right.$ : $\left.\llbracket M \rrbracket^{\mathbf{A}_{\omega}} \not \llbracket \llbracket \lambda x . x \rrbracket^{\mathbf{A}_{\omega}}\right\}$ is r.e. , so that the complement is co-r.e. We outline an algorithm to semi-decide $\left\{M \in \Lambda^{o}: \llbracket M \rrbracket^{\mathbf{A}_{\omega}} \not \llbracket \llbracket \lambda x . x \rrbracket^{\mathbf{A}_{\omega}}\right\}$ :

- Given $M \in \Lambda^{o}$, recursively enumerate the tokens of $\llbracket M \rrbracket^{\mathbf{A}_{\omega}}$ (this is possible by item (i)) and for each such token $\alpha$ run the algorithm that decides whether $\alpha \in \llbracket \lambda x . x \rrbracket^{\mathbf{A}_{\omega}}$ (this is possible by item (ii)); as soon as $\alpha \notin \llbracket \lambda x . x \rrbracket^{\mathbf{A}_{\omega}}$, stop and output "yes".

\section{THE MAIN THEOREM}

We say that a finite ris has cardinality $n \in \mathbb{N}$ if it has exactly $n$ tokens. We remark that every finite ris is isomorphic to a finite ris whose set of tokens is a finite subset of $\mathbb{N}$.

We now define an effective numeration $\left(\mathbf{E}_{m}\right)_{m \in \mathbb{N}}$ of all finite partial ris's whose tokens are natural numbers. Consider each natural number $m$ as the encoding of a triple $m=\left\langle n_{1}, n_{2}, n_{3}\right\rangle$, where $n_{1}$ codes a finite subset $X_{1} \subseteq \mathbb{N}, n_{2}$ codes a finite subset $X_{2} \subseteq \mathcal{P}_{\mathrm{f}}(\mathbb{N}) \times \mathcal{P}_{\mathrm{f}}(\mathbb{N})$, and $n_{3}$ codes a finite subset $X_{3} \subseteq \mathcal{P}_{\mathrm{f}}(\mathbb{N}) \times \mathcal{P}_{\mathrm{f}}(\mathbb{N}) \times \mathbb{N}$.

- If the pair $\mathcal{X}=\left(X_{1}, X_{2}\right)$ is an information system according to Definition 3.1 , and $X_{3}$ is the graph of a partial b-morphism $\rightarrow \mathrm{x}:(\mathcal{X} \Rightarrow \mathcal{X}) \rightarrow \mathcal{X}$, then we define $\mathbf{E}_{m}$ to be the finite ris $\mathbf{X}=(\mathcal{X}, \rightarrow \mathcal{X})$ defined by these data.

- Otherwise $\mathbf{E}_{m}$ is defined as the finite ris with empty web and completely undefined b-morphism. 
Recall that $\mathbf{E}_{m, \omega}$ is the completion of $\mathbf{E}_{m}$ and that $\mathbf{E}_{m, \omega}^{+}$, is a reflexive object in ALat, so that it has the structure of a $\lambda$-model. Therefore the Cartesian product $\mathbf{P}=\prod_{m \in \mathbb{N}} \mathbf{E}_{m, \omega}^{+}$is an algebraic lattice and a $\lambda$-model too (see Section 2.1), although it is not necessarily neither a reflexive object in ALat nor a filter model at all.

The following two lemmas are essential for carrying out Theorem 7.3.

Lemma 7.1. The order theory $\operatorname{Ord}(\mathbf{P})$ of the Cartesian product $\mathbf{P}$ is contained within the order theory of every ris-model. In particular, $\operatorname{Ord}(\mathbf{P})$ is contained within the order theory of every filter model which lives in $\mathbf{C p o .}$

Proof. By Theorem 5.6 every inequality $M \sqsubseteq N$, which fails in a ris-model, fails in the canonical completion of a finite ris, and then in $\mathbf{P}$ too, $\operatorname{since} \operatorname{Ord}(\mathbf{P})=$ $\bigcap_{k \in \mathbb{N}} \operatorname{Ord}\left(\mathbf{E}_{k, \omega}^{+}\right)$. By Section 4.1.2 every filter model $\mathbf{F}$ which lives in $\mathbf{C p o}$ is a ris-model, so that $\operatorname{Ord}(\mathbf{P}) \subseteq \operatorname{Ord}(\mathbf{F})$.

Lemma 7.2. For every non-trivial ris model $\mathbf{A}$ and every two closed distinct $\beta \eta$-normal forms $M, N$ we have $\llbracket M \rrbracket^{\mathbf{A}} \nsubseteq \llbracket N \rrbracket^{\mathbf{A}}$.

Proof. Let $\mathbf{A}$ be a non-trivial ris model and let $M, N$ be closed distinct $\beta \eta$-normal forms. By Theorem 2.3 there exists a sequence $\vec{L}$ of $\lambda$-terms such that $M \vec{L}={ }_{\beta}$ $\lambda x y . y$ and $N \vec{L}={ }_{\beta} \lambda x y . x$; note that the interpretations $\llbracket M \vec{L} \rrbracket^{\mathbf{A}}$ and $\llbracket N \vec{L} \rrbracket^{\mathbf{A}}$ do not depend on any environment. Now assuming, by contradiction, that $\llbracket M \rrbracket^{\mathbf{A}} \subseteq \llbracket N \rrbracket^{\mathbf{A}}$ and using the monotonicity of application in $\mathbf{A}$ we obtain that for arbitrary $a, b \in A$ it holds that $a=\llbracket \lambda x y \cdot x \rrbracket^{\mathbf{A}} a b=\llbracket M \vec{L} \rrbracket^{\mathbf{A}} a b \subseteq \llbracket N \vec{L} \rrbracket^{\mathbf{A}} a b=\llbracket \lambda x y \cdot y \rrbracket^{\mathbf{A}} a b=b$. This contradicts the non-triviality of $\mathbf{A}$.

Theorem 7.3. There exists a non-empty co-r.e. set of easy terms that are not simple easy.

Proof. We claim that the set $X=\left\{N \in \Lambda^{o}: \llbracket N \rrbracket^{\mathbf{P}} \leq \llbracket \lambda x . x \rrbracket^{\mathbf{P}}\right\}$ is non-empty, $\beta$-closed and co-r.e. . Non-emptyness and $\beta$-closure of $X$ are trivial. For the last point, we show that the complement of $X$ is r.e. .

We define a binary relation $R$ as follows:

$$
R(M, m) \Longleftrightarrow \llbracket M \rrbracket^{\mathbf{E}_{m, \omega}} \nsubseteq \llbracket \lambda x \cdot x \rrbracket^{\mathbf{E}_{m, \omega}}
$$

Recall by Lemma 6.3(i-ii) that, for every $m$, the set $\llbracket \lambda x \cdot x \rrbracket^{\mathbf{E}_{m, \omega}}$ is decidable and that, for every $m$ and $M$, the set $\llbracket M \rrbracket^{\mathbf{E}_{m, \omega}}$ is r.e. . Note that by Lemma 7.2 $R(\lambda x y . x y, m)$ holds for all $m$.

Now we define an algorithm:

$$
\text { Interpretation }(M, m, l)
$$

with the following specification:

- takes as input a $\lambda$-term $M$ and two numbers $m, l$ and performs $l$ steps of the completion of $\mathbf{E}_{m}$. Call $\mathbf{B}$ the result of this partial completion. Since $\llbracket \lambda x . x \rrbracket^{\mathbf{E}_{m, \omega}}$ is decidable and $\llbracket M \rrbracket^{\mathbf{B}}$ is finite, then the algorithm outputs the pair $(M, m)$, if $\llbracket M \rrbracket^{\mathbf{B}} \nsubseteq \llbracket \lambda x . x \rrbracket^{\mathbf{E}_{m, \omega}}$, and the pair $(\lambda x y . x y, m)$ otherwise. 
The range of outputs of Interpretation $(M, m, l)$ is exactly the predicate $R$. Therefore the relation $R(M, m)$ is r.e. and hence so is the predicate $\exists m . R(M, m)$. We conclude that $\Lambda^{o}-X$ is r.e. since $M \in \Lambda^{o}-X \Longleftrightarrow \exists m . R(M, m)$.

By Theorem 2.1 the intersection of the non-empty $\beta$-closed co-r.e. set $X$ and of the non-empty $\beta$-closed co-r.e. set $E$ of all easy terms is co-r.e. and nonempty. Let $M \in X \cap E$. Assume, by contraposition, $M$ to be simple easy, so that by Theorem 2.9 there exists a non-trivial filter model $\mathbf{F}$ in the category $\mathbf{C p o}$ such that $\llbracket M \rrbracket^{\mathbf{F}}=\llbracket \lambda x y \cdot x \rrbracket^{\mathbf{F}}$. From $M \in X$ it follows that $\llbracket M \rrbracket^{\mathbf{P}} \leq \llbracket \lambda x . x \rrbracket^{\mathbf{P}}$; therefore, by Lemma 7.1 we obtain that $\llbracket M \rrbracket^{\mathbf{F}} \leq \llbracket \lambda x . x \rrbracket^{\mathbf{F}}$. Finally we obtain $\llbracket \lambda x y . x \rrbracket^{\mathbf{F}}=\llbracket M \rrbracket^{\mathbf{F}} \leq \llbracket \lambda x . x \rrbracket^{\mathbf{F}}$ and this contradicts Lemma 7.2. We conclude that $M$ is easy (since $M \in E$ ) but not simple easy.

\section{REFERENCES}

[1] F. Alessi and M. Dezani-Ciancaglini. TLCA list of open problems: Problem 19, http://tlca.di.unito.it/opltlca/problem19.pdf, 2002.

[2] F. Alessi, M. Dezani-Ciancaglini, and F. Honsell. Filter models and easy terms. In ICTCS '01: Proceedings of the 7th Italian Conference on Theoretical Computer Science, pages 17-37, London, UK, 2001. Springer-Verlag.

[3] F. Alessi, M. Dezani-Ciancaglini, and S. Lusin. Intersection types and domain operators. Theor. Comput. Sci., 316:25-47, May 2004.

[4] F. Alessi and S. Lusin. Simple easy terms. Electronic Notes in Theoretical Computer Science, 70(1):1 - 18, 2003. ITRS '02, Intersection Types and Related Systems (FLoC Satellite Event).

[5] J.C.M. Baeten and B. Boerboom. Omega can be anything it should not be. Indagationes Mathematicae (Proceedings), 82(1):111 - 120, 1979.

[6] H.P. Barendregt. Some extensional term models for combinatory logics and $\lambda$-calculi. $\mathrm{PhD}$ thesis, University of Utrecht, 1971.

[7] H.P. Barendregt. The Lambda calculus: Its syntax and semantics. North-Holland, Amsterdam, 1984.

[8] H.P. Barendregt, M. Coppo, and M. Dezani-Ciancaglini. A filter lambda model and the completeness of type assignment. The Journal of Symbolic Logic, 48(4):pp. 931-940, 1983.

[9] O. Bastonero, A. Pravato, and S. Ronchi Della Rocca. Structures for lazy semantics. In Gries and de Roever, editors, Programming Concepts and Methods (PROCOMET'98), pages 30 - 48. Chaptman \& Hall, 1998.

[10] A. Berarducci. Infinite lambda-calculus and non-sensible models. In Logic and Algebra (Pontignano 1994) (Lecture Notes in Pure and Applied Mathematics Series/180), pages 339-378. Marcel Dekker Inc., 1996.

[11] A. Berarducci and B. Intrigila. Some new results on easy lambda-terms. Theoretical Computer Science, 121(1-2):71 - 88, 1993.

[12] C. Berline. From computation to foundations via functions and application: The $\lambda$-calculus and its webbed models. Theor. Comput. Sci., 249:81-161, October 2000.

[13] C. Berline. Graph models of $\lambda$-calculus at work, and variations. Mathematical. Structures in Comp. Sci., 16:185-221, April 2006.

[14] C. Berline, G. Manzonetto, and A. Salibra. Effective $\lambda$-models versus recursively enumerable $\lambda$-theories. Mathematical. Structures in Comp. Sci., 19:897-942, October 2009.

[15] C. Berline and A. Salibra. Easiness in graph models. Theor. Comput. Sci., 354:4-23, March 2006.

[16] G. Berry. Stable models of typed lambda-calculi. In ICALP'ry, LNCS 62, Berlin, 1978. Springer-Verlag. 
[17] C. Böhm. Alcune proprietá delle forme $\beta$ - $\eta$-normali nel $\lambda$-K-calcolo. Technical Report 696, CNR, 1968.

[18] A. Bucciarelli and T. Ehrhard. Sequentiality and strong stability. In LICS'91, pages 138-145. IEEE Computer Society Press, 1991.

[19] A. Carraro and A. Salibra. Reflexive Scott domains are not complete for the extensional lambda calculus. In Proceedings of the Twenty-Fourth Annual IEEE Symposium on Logic in Computer Science (LICS 2009), pages 91-100. IEEE Computer Society Press, August 2009.

[20] M. Coppo and M. Dezani-Ciancaglini. An extension of the basic functionality theory for the $\lambda$-calculus. Notre-Dame Journal of Formal Logic, 21(4):685-693, October 1980.

[21] M. Coppo, M. Dezani-Ciancaglini, F. Honsell, and G. Longo. Extended type structures and filter lambda models. In G. Lolli, G. Longo, and A. Marcja, editors, Logic Colloquium 82, pages 241-262, Amsterdam, the Netherlands, 1984. North-Holland.

[22] M. Coppo, M. Dezani-Ciancaglini, F. Honsell, and G. Longo. Extended type structures and filter lambda models. In G. Lolli, G. Longo, and A. Marcja, editors, Logic Colloquium 82, pages 241-262, Amsterdam, the Netherlands, 1984. North-Holland.

[23] E. Engeler. Algebras and combinators. Algebra Universalis, 13(3):389-392, 1981.

[24] F. Honsell and S. Ronchi Della Rocca. Reasoning about interpretations in qualitative lambda models. In Proceedings of the IFIP Working Group 2.2/2.3, pages 505-521. M. Broy and C.B. Jones editors, North-Holland, 1990.

[25] F. Honsell and S. Ronchi Della Rocca. An approximation theorem for topological lambda models and the topological incompleteness of lambda calculus. Journal of Computer and System Sciences, 45:49-75, 1992.

[26] B. Intrigila. A problem on easy terms in lambda-calculus. Fundamenta Informaticae, 15(1):99-106, 1991.

[27] G. Jacopini. A condition for identifying two elements of whatever model of combinatory logic. In Proceedings of the Symposium on Lambda-Calculus and Computer Science Theory, pages 213-219, London, UK, 1975. Springer-Verlag.

[28] G. Jacopini and M. Venturini-Zilli. Equating for recurrent terms of $\lambda$-calculus and combinatory logic. Technical Report 85, CNR, 1978.

[29] G. Jacopini and M. Venturini-Zilli. Easy terms in the lambda-calculus. Fundamenta Informaticae, 8(2):225-233, 1985.

[30] R. Kerth. Isomorphism et équivalence équationnelle entre modèles du $\lambda$-calcul. Master's thesis, Université de Paris 7, 1995. Thèse.

[31] R. Kerth. Isomorphism and equational equivalence of continuous lambda models. Studia Logica, 61:403-415, 1998.

[32] R. Kerth. On the construction of stable models of $\lambda$-calculus. TCS, 269:23-46, 2001.

[33] C.P.J. Koymans. Models of the lambda calculus. Information and Control, 52(3):306-332, 1982.

[34] J. Kuper. On the Jacopini technique. Information and Computation, 138(2):101 - 123, 1997.

[35] G. Longo. Set-theoretical models of $\lambda$-calculus: theories, expansions, isomorphisms. Ann. Pure Appl. Logic, 24(2):153-188, 1983.

[36] A.R. Meyer. What is a model of the lambda calculus? Information and Control, 52(1):87$122,1982$.

[37] G. D. Plotkin. Set-theoretical and other elementary models of the $\lambda$-calculus. Theor. Comput. Sci., 121(1-2):351-409, 1993.

[38] A. Salibra. Topological incompleteness and order incompleteness of the lambda calculus. ACM Trans. Comput. Logic, 4:379-401, July 2003.

[39] D.S. Scott. Continuous lattices. In Toposes, algebraic geometry and logic, Berlin, 1972. Springer-Verlag.

[40] D.S. Scott. Data types as lattices. In Gert Müller, Arnold Oberschelp, and Klaus Potthoff, editors, ISILC Logic Conference, volume 499 of Lecture Notes in Mathematics, pages 579651. Springer Berlin / Heidelberg, 1975. 10.1007/BFb0079432. 
[41] D.S. Scott. Lambda calculus: some models, some philosophy. In The Kleene Symposium, Amsterdam, The Netherlands, 1980. North-Holland.

[42] D.S. Scott. Domains for denotational semantics. In ICALP '82, pages 95-104, New York, NY, USA, 1982. ACM Press.

[43] A. Visser. Numerations, $\lambda$-calculus, and arithmetic. In J.R. Hindley and J.P. Seldin, editors, To H. B. Curry: Essays on Combinatory Logic, Lambda Calculus and Formalism, pages 259-284, 1980.

[44] C. Zylberajch. Syntaxe et sémantique de la facilité en $\lambda$-calcul. Thèse de doctorat d'état, Université Paris 7, 1991.

Communicated by (The editor will be set by the publisher).

(The dates will be set by the publisher). 